\title{
Biomechanical constitutive modeling of the gastrointestinal tissues: where are we?
}

\author{
Bhavesh Patel $^{\mathrm{a}}$, Alessio Gizzi ${ }^{\mathrm{b}}, \mathrm{Javad}$ Hashemi $^{\mathrm{a}}$, Yousif Awakeem ${ }^{\mathrm{a}}$, Hans Gregersen ${ }^{\mathrm{a}}$, \\ Ghassan Kassab ${ }^{\mathrm{a}, *}$ \\ ${ }^{a}$ California Medical Innovations Institute, 11107 Roselle St, San Diego, CA 92121, USA \\ ${ }^{b}$ Department of Engineering, Campus Bio-Medico University of Rome, Via A. del Portillo 21, 00128 \\ Rome, IT
}

\begin{abstract}
The gastrointestinal (GI) tract is a continuous channel through the body that consists of the esophagus, the stomach, the small intestine, the large intestine, and the rectum. Its primary functions are to move the intake of food for digestion before storing and ultimately expulsion of feces from the rectum through the anal sphincter. The mechanical behavior of GI tissues thus plays a crucial role for GI function in health and disease. The mechanical properties are typically characterized by a constitutive biomechanical model, which is a mathematical representation of the relation between load and deformation in a tissue. Hence, validated biomechanical constitutive models are essential to characterize and simulate the mechanical behavior of the GI tract under physiological and pathological conditions. Numerous constitutive models have consequently been proposed over the past three decades, mainly inspired by work done in cardiovascular tissues. Here, a comprehensive review of these constitutive models is provided. This review is limited to studies where a model of the strain energy function is proposed to characterize the stress-strain relation of a GI tissue. Several needs are identified for more advanced modeling of GI biomechanics including: 1) Microstructural models that provide actual structure-function relations; 2) Validation of coupled electro-mechanical models accounting for active muscle contractions; 3) Human data under physiological and pathological conditions to develop and validate models. The findings from this review provide guidelines for using existing constitutive models as well as perspective and directions for future studies aimed at
\end{abstract}

\footnotetext{
${ }^{*}$ Corresponding author

Email address: gkassab@calmi2.org, Tel: 001-858-249-7400, Fax: 001-858-249-7419 (Ghassan Kassab)
} 
establishing new constitutive models for GI tissues.

Keywords: Digestive tract, Colon, Biomechanics, Mechanical properties, Strain energy function, Hyperelasticity

\section{Introduction}

The gastrointestinal (GI) tract is a long conduit extending from the oral cavity to the anus and consists of five major segments: the esophagus, the stomach, the small intestine, the large intestine, and the rectum (Figure 1). Each segment is separated by sphincters

5 such as the lower, esophageal sphincter, pylorus, and anal sphincters. Although there are anatomical similarities between these segments of the GI tract, there are also differences and in particular differences in function. The GI tract acts as a digestive mechanical system that transforms food into chyme and ultimately feces during transit trough the GI system. Healthy functioning of the GI tract is crucial for absorbing essential nutrients 10 (carbohydrates, proteins, fats, water, minerals, and vitamins) from food as well as for secretion of chemical substances that aid breakdown of the food constituents [1]. Most drugs are administered orally, and their efficacy also relies on the healthy functioning of the GI 2. The GI functions are affected by numerous structural and functional GI diseases and disorders such as gastro-esophageal reflux disease (GERD), dyspepsia, 15 irritable bowel syndrome (IBS), diverticulosis, constipation, diarrhea, hemorrhoids, and fecal incontinence. These diseases and disorders have a significant impact on quality of life and impose a major financial burden on patients and healthcare systems [3 9 . Unfortunately, these GI diseases and disorders are highly prevalent with functional GI diseases alone affecting over $40 \%$ of the world population [10].

Most of these diseases and disorders are invariably linked to altered GI biomechanics [11 13. Studying the deformation of GI organs and the forces that causes such deformation is thus crucial for understanding their mechanical behavior in health and disease. The mechanical properties are also essential for developing computational models of GI organs that can generate hypotheses, support diagnosis, predict treatment outcomes, and virtually evaluate new therapeutic approaches. Mathematically, deformation is typically quantified in terms of strain, which is a measure of stretch relative to a reference state, while force is quantified in terms of stress, which is a measure of force per unit surface 
area. A mechanical constitutive model is a mathematical formulation that relates stress and strain in a material through parameters 14. It is essential for characterizing the mealso constitutes one of the three major elements of biomechanical computational models, along with tissue geometry and boundary conditions.

In the present work, we provide a comprehensive review of the published constitutive models of the five GI organs (GI sphincters were excluded). The focus is on studies where has been shown to capture well the non linear and large deformation of soft tissues, including GI tissues. The GI tract consists of four main layers all with different mechanical characteristics: submucosa, mucosa, muscle, and serosa. In the review, we consider studies that model intact GI tissue and/or any combination of the individual layers. Over the last 40 three decades, numerous strain-energy derived constitutive models have been proposed for the GI tissues, primarily inspired by models and methods validated in cardiovascular tissues. After summarizing the most common models and their formulations, we conduct a cross-analysis of all the studies comparing them based on several factors such as organ studied, model type, testing protocol followed, species used for the tissue, etc. The objectives are two-fold: 1) Provide an overview of the validated constitutive models of GI tissues currently established to assist in the selection of a suitable model and 2) Identify gaps in knowledge to provide guidelines for future development of constitutive models.

The review strategy is described in Section 2 and the availability of data is discussed in Section 3 . Section 4 provides a brief overview of the constitutive modeling process.

${ }_{50}$ Subsequently, a review of passive and active constitutive models of GI tissues is given in Section 5 and Section 6 respectively. In Section 7 , the results from our cross-analysis are presented. Section 8 ends the review with a discussion of the open challenges, perspectives, and directions for future studies based on our findings. A summary of the notation used in the manuscript is provided in Appendix A.

\section{2. Review strategy}

A PubMed search was performed for each GI organ using the terms listed in Table S1 (supplemental material, c.f. Section 3). The search was set to Title/Abstract. An 
upper bound limit of April $30^{\text {st }}, 2021$ (included) was set for the publication date. No lower bound limit was specified. Additional manuscripts were included based on the authors' knowledge if they were not encountered during the search. The search was conducted in several iterations and ended during May 2021. After screening relevant titles and abstracts, the manuscripts were read to confirm eligibility. Our eligibility criteria consisted of retaining only English language articles where new material parameters are derived for the first time, using new or previously published mechanical test data, for a newly or previously formulated model of the strain energy function for the tissue of interest. A total of 49 manuscripts were found to meet these criteria and were retained for analysis in this work. Three of these manuscripts studied both the large intestine and the rectum. One manuscript studied the small intestine, the large intestine, and the rectum. All the studies were counted individually for each organ to simplify the analysis, thus leading to a total count of 54 studies. The distribution of these studies over the different GI organs is shown in Fig. 1. The details of the review process are provided in the PRISMA diagram given in Fig. 2.

\section{Data availability}

All the data associated with this manuscript is openly available in a dataset archived on 75 Zenodo [15]. Multiple information was extracted from the studies to conduct our analysis, including title, publication year, species for the source of the tissue, test protocol, model category, etc. All results from the review have been stored in a spreadsheet called "reviewsummary.xlsx". The spreadsheet also includes a glossary of the technical terms used to characterize and classify the different studies in this work. We refer to that glossary for most of the classification terminology used in this manuscript. A Jupyter notebook called "post-processing-code.ipynb" has been prepared to analyze the results and generate the figures of interest. Results and figures deemed most relevant are included in this paper. Readers are invited to view the spreadsheet as well as use the Jupyter notebook for further analysis if desired. The dataset also includes our Supplemental Material file (supplemental.pdf) that contains the sections and the tables referred to in this paper (they include "S" in their label). We have followed applicable FAIR data standards and made all data available to anyone without restrictions [16. Specifically, the dataset was organized 
according to the SPARC Data Structure using the SPARC data curation software SODA [17 19] and the files are made available under open and permissive licenses (CC-BY for data files and MIT for code files).

\section{Basis of constitutive modeling}

We provide here an overview of the constitutive modeling process. This section is limited to concepts identified during our review of GI tissues although it could be applicable beyond. For more details related to continuum mechanics or constitutive modeling of soft 95 tissues, we refer the readers to the literature available on these topics $20-22]$.

\subsection{Strain energy function}

It has been shown that soft tissues can endure large deformation and their non-linear load-displacement behavior is best modeled by defining a Helmholtz free strain energy function $\Psi$ such that the stress and strain in the material are related as follows:

$$
\boldsymbol{\sigma}=\frac{2}{J} \mathbf{F} \frac{\partial \Psi}{\partial \mathbf{C}} \mathbf{F}^{T}
$$

where $\boldsymbol{\sigma}$ is the true Cauchy stress tensor, $\mathbf{F}$ is the deformation gradient, $J=\operatorname{det}(\mathbf{F})$ refers to the Jacobian of the deformation map, and $\mathbf{C}=\mathbf{F}^{T} \mathbf{F}$ designates the right Cauchy-Green deformation tensor. The constitutive modeling process consists of establishing an explicit formulation of $\Psi$, as explained in the next section. The formulations established for GI tissues are discussed in detail in Sections 5 and 6 . Here, we provide an overview of the structure of $\Psi$.

GI tissues are modeled as homogeneous material, meaning that $\Psi$ is independent of the location on the tissue. This has been deemed as an acceptable assumption for all soft tissues at the scale of interest. Moreover, a decoupled form is assumed for $\Psi$ such that:

$$
\Psi=U(J)+W
$$

where $U$ is a purely volumetric (dilatational) contribution and $W$ is a purely isochoric (volume preserving) contribution. The volumetric term $U$ is only considered in a few of the studies reviewed 23 28]. In most studies, GI tissues are typically assumed to be incompressible (i.e., their volume remains constant). Interestingly, no GI-specific studies 
are referenced to support this assumption. It appears to have been extrapolated from studies on other soft tissues. Under the incompressibility assumption, $U=0$. Since $J$ represents the change in volume with respect to the reference configuration, we also have $J=1$. The incompressibility constraint $J-1=0$ is typically imposed directly on the energy function as:

$$
\Psi=W-p(J-1)
$$

Here, $p$ serves as an indeterminate Lagrange multiplier to enforce incompressibility. It can be identified as the hydrostatic pressure and may only be determined from the equilibrium equations and the boundary conditions. For GI tissues, the isochoric contribution $W$ is typically split as follows:

$$
W=W^{p}+W^{a}
$$

where $W^{p}$ represents the passive contribution and $W^{a}$ the active contribution. The passive contribution is associated with the mechanical contribution of fibers (elastin, collagen) in the tissue and the ground matrix embedding these fibers while the active contribution is associated with the contraction of the smooth and striated muscle cells and the electrical behavior of pacemaker cells.

\subsection{Modeling of passive mechanical properties}

A majority of the studies reviewed investigated passive behavior only. The passive contribution $W^{p}$ of the strain energy function can be further split as follows:

$$
W^{p}=W^{e}+W^{v}
$$

Here, $W^{e}$ represents the hyperelastic part that is associated with the instantaneous response of the tissue and is only dependent on the strain. $W^{v}$ represents the viscous part associated with the rearrangement mechanisms of tissue micro-components over time. This component is observable at the tissue level through stress relaxation at constant strain, creep at constant stress, hysteresis during loading and unloading, and strain-rate dependence. Most studies of GI tissues have only investigated passive hyperelastic models for formulating $W^{e}$ (c.f. section 5.1). In this review, we have classified them into three categories:

1. Phenomenological models: Do not explicitly account for tissue structure; 
2. Structure-based models: Only average orientation of the fibers and their overall mechanical contribution are accounted in the strain energy function;

3. Microstructure-based models: Strain energy function accounts for the mechanical behavior of the individual fibers and their orientation.

Some studies have investigated the passive visco-hyperelastic behavior of GI tissues and established a model of the strain energy function that includes both the hyperelastic and viscous contributions (c.f. section 5.2.

\subsection{Modeling of active mechanical properties}

Only select studies have investigated active constitutive models for capturing the complex active behavior of GI tissues linked to the electrical pacemaking activity of the Interstitial Cells of Cajal (ICC) with respect to smooth muscle contraction (c.f. section 6 ). Some studies consider the full tissue behavior including both passive and active contributions while others focus only on the active part. Some studies derive a model for the active part $W^{a}$ of the strain energy function. Others consider different approaches to account for the active contribution through the inclusion of an active stress or active strain component. In this review, we have classified active models into three categories:

1. Active stress-based: The total equilibrium stress in the tissue results from the superposition of a passive and an active contribution;

2. Active strain-based: A multiplicative decomposition of the deformation gradient is assumed to introduce active distortions generating material contractility;

3. Active electromechanics-based: An additive decomposition of the strain energy is assumed to enforce active and passive stress contributions accounting for electric field and material distortions.

\subsection{Typical constitutive modeling process}

Constitutive modeling in biomechanics consists of identifying a suitable mathematical model/formulation of the strain energy function $\Psi$ for the tissue of interest [29]. Numerous standard formulations cover the diverse range of behavior of soft tissues, as we will summarize in the next sections. Each of these formulations contains unknown parameters, commonly designated as material parameters. If an optimal tuning of these 
parameters is performed for a given model of the strain energy function, such as to capture the actual stress-strain relation of a tissue, then it is considered a suitable constitutive model for the tissue. To this aim, the constitutive modeling process, independently of the multiphysics behavior involved, typically starts with mechanical testing on the tissue of interest. During the mechanical test, measures of force (axial force, pressure, etc.), related deformation (axial stretch, change in radius, etc.), and/or external stimuli (boundary constraints, electric field, voltage, current, etc.) are simultaneously recorded. The shape of the deformation tensor $\mathbf{F}$ is defined based on the test type, and components of $\mathbf{F}$ are calculated for each imposed/measured deformation during the test. A mathematical formulation, i.e., constitutive model, is then established for $\Psi$, relying typically on the known structure of the tissue and/or previous work. The Cauchy stress in Equation 1 is then only a function of the material parameters of the selected constitutive model. To estimate these parameters, either the experimentally measured force quantities are converted to corresponding stress values or theoretical formulations are established for these quantities as a function of the Cauchy stress components. As an alternative to theoretical formulations, computational estimations based on the Finite Element Method (FEM) are also used in some studies [30, 31. Then, optimal material parameters of $W$ for the tissue are estimated by fitting the theoretical/computational stress/force values to the corresponding experimental ones for each experimentally imposed/measured deformation. During the fitting process, the following cost function is typically minimized numerically as:

$$
e(\mathbf{s})=\sum_{i=1}^{N}\left(A_{i}^{e x p}-A_{i}^{\text {mod }}(\mathbf{s})\right)
$$

where, $N$ indicates the number of experimental data points, $\mathbf{s}$ refers to the set of material parameters for the strain energy function of choice, "exp" designates experimental values from mechanical tests, and "mod" indicates the corresponding theoretical/computational value based on the selected constitutive model and associated material parameters. The symbol $A$ represents the quantity that is fitted, which depends on the type of mechanical tests conducted. For instance, $A$ is the axial Cauchy stress $\sigma_{a}$ if only uniaxial tests were conducted and the cost function is expressed as:

$$
e(s)=\sum_{i=1}^{N}\left(\sigma_{a, i}^{e x p}-\sigma_{a, i}^{\bmod }(s)\right)
$$


Parameters quantifying the goodness of fit (e.g., the final cost function value $e(s)$, the coefficient of determination $R^{2}$, and the reduced chi-square $\chi$ ) are finally computed to decide if the selected strain energy function is suitable for the tissue. If so, the parameters are commonly validated with test data not used for parameter optimization to confirm the suitability of the strain energy function. If not, the process is repeated with another choice for the constitutive model until a satisfactory model is identified [32, 33. Once a suitable model is identified and the material parameters are estimated, an explicit relation is obtained between the stress and strain in the tissue. The formulation can then be used to analyze the mechanical characteristics of the tissue or integrated into a computational framework, e.g., using the FEM, to conduct load/deformation simulation of the tissue.

In the GI studies reviewed, different constitutive models and constitutive modeling processes (mechanical testing protocol, tissue source, etc.) have been proposed. They are discussed in the subsequent sections.

\section{Passive models of GI tissues}

We provide in this section an overview of the most common formulation for passive GI tissues encountered during our review.

\subsection{Hyperelastic models}

\subsubsection{Phenomenological models}

The first model of a hyperelastic strain energy function applied to a GI tissue was Fung's model by Miftakhov and colleagues in their study of the small intestine published in 1994 [34]. In fact, Fung's model was used in the first six studies of hyperelastic constitutive modeling of GI tissues [34-39]. It has subsequently been utilized in numerous GI tissue studies [12, 40,47. A list of all such studies is provided in Table S2 along with relevant information. It has been found to characterize well the behavior of the intact tissue of all five GI organs as well as the individual layers of the esophagus. Validation for GI tissues has been achieved against uniaxial tests (planar uniaxial extension and tubular inflation), biaxial tests (planar biaxial extension and tubular inflation-extension), and triaxial tests (tubular inflation-extension-torsion).

Fung's model was originally introduced to characterize arterial tissues [48 52]. It is developed in terms of the components $E_{i j}$ of the Green-Lagrange strain tensor $\mathbf{E}=\frac{1}{2}(\mathbf{C}-\mathbf{I})$ 
with $\mathbf{C}=\mathbf{F}^{T} \mathbf{F}$ being the right Cauchy-Green deformation tensor. It is expressed in its most general form as follows:

$$
W=\frac{c}{2}\left(e^{Q}-1\right), \quad Q=a_{i j k l} E_{i j} E_{k l}, \quad i, j, k, l=r, \theta, z
$$

The constant $c$ is a stress-like material parameter while $a_{i j k l}$ are dimensionless material parameters, and the notation $r, \theta, z$ refer to the radial, circumferential, and longitudinal directions associated with the tubular tissue of interest. None of these parameters relate to the structure of the tissue, and hence the phenomenological nature of this model. In most studies, the thickness of the tissue is assumed to be negligible compared to the radius and the length leading to a plane stress assumption in the radial direction. Moreover, the tissue behavior is assumed to be orthotropic and shear is not considered. These assumptions lead to the following three exponential parameters formulation [12, 36, 37, 40, 41, 43, 45, 47]:

$$
Q=a_{\theta \theta \theta \theta} E_{\theta \theta}^{2}+a_{z z z z} E_{z z}^{2}+2 a_{\theta \theta z z} E_{\theta \theta} E_{z z}
$$

Such a model was previously proposed for capturing the behavior of arteries [49, 53], myocardium [54], and epicardium [55], among others. Other studies have considered nonzero stress along the thickness of the tissue [35, 42], shear in the $\theta-z$ plane [38, 39], or both [47], leading to a higher number of exponential parameters. Three studies have proposed a formulation with an additive quadratic term to Fung's model [34, 40, 46]:

$$
W=\frac{c}{2}\left(e^{Q}-1\right)+\frac{1}{2} q, \quad q=b_{i j k l} E_{i j} E_{k l}, \quad i, j, k, l=r, \theta, z
$$

170 Such a model was previously proposed for characterizing the behavior of skin [56], arteries [57. 58], visceral pleura [59], and pericardium [60], among others. The term $q$, referred to in some studies as the quadratic component, characterizes the tissue's linear behavior (typically at small strains), while the exponential term dictates the non-linear behavior (at larger strains). All three GI studies mentioned above used a three-parameter $\left(b_{\theta \theta \theta \theta}\right.$,

$175 b_{z z z z}$, and $\left.b_{\theta \theta z z}\right)$ quadratic term [34, 40,46]. In one of these studies, it was found that the model with a three-parameter quadratic term and three-parameter exponential Fung term provided a good fit against tubular inflation-extension loading in all the ranges tested for the small intestine tissue while the three and six exponential parameter Fung models without quadratic term only provided a good fit against data in physiological loading ranges [46]. In another study [40], it was shown that use of a three-parameter 
quadratic term with a three-parameter exponential Fung term did not provide a better fit than using a three-parameter exponential Fung term alone for the mucosa-submucosa and muscle layers of the esophagus. In the same study, the authors also tested a neoHookean model (c.f. next paragraph) instead of the quadratic term and reached the same conclusion.

Various other phenomenological models have also been proposed to describe the hyperelastic mechanical behavior of intact stomach, large intestine, and rectum tissues as well as the individual layers of the large intestine. A list of such studies is provided in Table S3 along with relevant information. Contrary to Fung's model, these other phenomenological models are isotropic and thus do not account for the variation of tissue behavior depending on the loading/deformation direction. These models have only been validated against tissue testing results in one direction at a time using compression and/or planar uniaxial tests. Among these, invariant-based phenomenological models have been most commonly utilized. They take the form of a polynomial function in the two invariants $I_{1}=\operatorname{tr}(\mathbf{C})$ and $I_{2}=\frac{1}{2}\left(\operatorname{tr}(\mathbf{C})^{2}+\operatorname{tr}\left(\mathbf{C}^{2}\right)\right)$ of the right Green-Cauchy deformation tensor C as:

$$
W=\sum_{i, j=0}^{n} c_{i j}\left(I_{1}-3\right)^{i}\left(I_{2}-3\right)^{j}
$$

The constants $c_{i j}$ are the material parameters (with $c_{00}=0$ ) with unit of pressure. In the particular case where only $c_{10}$ is non-zero, the strain energy function is designated as the neo-Hookean model [61]. In the case where only $c_{10}$ and $c_{01}$ are non-zero, it is referred to as the Mooney-Rivlin model [62, 63. One study proposed an extended Mooney-Rivlin model for the large intestine tissue where $c_{11}$ is also non-zero [64]. One study also used a 2nd order reduced polynomial ( $c_{10}$ and $c_{20}$ non-zero) for modeling the individual behavior of the mucosa, submucosa, and muscle layers of the stomach. It is worth noting that in one study where Fung's, Neo-Hookean, and Mooney-Rivlin models were tested, it was found that Fung's model reproduced best the planar biaxial behavior of the small intestinal tissue while Neo-Hookean and Mooney-Rivlin performed very poorly [43].

Besides invariant-based models, Ogden's model [65], which depends on the principal stretches $\lambda_{i}$ with $i=r, \theta, z\left(\lambda_{i}^{2}=\operatorname{eigen}(\mathbf{C})\right)$, has been used in various studies [66 69] as 
listed in Table S3. The general form of the Ogden's model is expressed as follows

$$
W=\sum_{k=1}^{N} \frac{\mu_{k}}{\alpha_{k}}\left(\lambda_{r}^{\alpha_{k}}+\lambda_{\theta}^{\alpha_{k}}+\lambda_{z}^{\alpha_{k}}-3\right)
$$

Here, $\mu_{k}$ (unit of pressure) and $\alpha_{k}$ (dimensionless) are the material parameters of the model. The constant $N$ determines the order of the model. For GI tissues, $N=1$ $[66,67,69]$ and $N=3[68]$ have been used.

\subsubsection{Structure-based models}

200 Most of the structure-based models for GI tissues are inspired by the formulation, and its variants, proposed by Holzapfel, Gasser, Ogden, and collaborators for arterial tissues $[70-72]$. We refer to them here as Holzapfel-type models. These models account for the fiber orientation in the tissue and hence their classification as structure-based. A Holzapfel-type model was the second type ever to be tested (after Fung's type model) for

a GI tissue in two studies by Yang and colleagues on the esophagus published in 2006 [73, 74, followed by numerous studies subsequently [28, 75, 81]. A list of such studies is provided in Table S4 along with relevant information. Overall, Holzapfel-type models characterize well the mechanical behavior of the intact tissue of the esophagus, small intestine, large intestine, and rectum as well as the individual layers of the esophagus, stomach, large intestine, and rectum. Validation has been achieved against uniaxial tests (planar uniaxial extension, planar shear) and biaxial tests (planar biaxial extension and tubular inflation-extension)

An additive split of the strain-energy function is suggested in Holzapfel-type models between a part associated with isotropic deformations and a part associated with anisotropic contribution, as follows

$$
W=W_{\text {iso }}+W_{\text {aniso }}
$$

The isotropic contribution is associated with the mechanical response of the non-collagenous components of the tissue (matrix material, elastin) [27, 70]. The use of the neo-Hookean model is typically prescribed as

$$
W_{\text {iso }}=C_{10}\left(I_{1}-3\right)
$$


In one study for the esophagus, no isotropic part was included [76]. Rather, the noncollageneous part was attributed solely to the elastin fibers, and an anisotropic pseudoelastic model was used to describe its behavior. The anisotropic contribution is associated with the mechanical response of collagenous fibers. In its most general form, an exponential function of the following form is used to describe the strain energy stored in the collagen fibers of the tissue:

$$
W_{\text {aniso }}=\sum_{j}^{N} W_{\text {aniso }}^{(j)}=\sum_{j}^{N} \frac{k_{1}^{(j)}}{n k_{2}^{(j)}}\left[e^{Q^{(j)}}-1\right]
$$

with

$$
Q^{(j)}=\left\{\begin{array}{c}
k_{2}^{(j)}\left(I_{4}^{(j)}-1\right)^{2} \quad \text { if } \quad I_{4}^{(j)} \geq 1 \\
0 \text { otherwise }
\end{array}\right.
$$

Here, the superscript $j$ designates a family of collagen fibers, typically defined as all collagen fibers aligned along the same mean direction. The coefficient $N$ indicates the total number of fiber families and $n$ is typically equal to $N$. The stress-like parameter $k_{1}^{(j)}>0$ and the dimensionless parameter $k_{2}^{(j)}>0$ describe the mechanical behavior of the fiber family $j$ at small and large strain, respectively, and are estimated using mechanical testing data. The anisotropic fourth invariant of the deformation $I_{4}^{(j)}$ is a measure of the stretch of the fibers from family $j$. Because of the wavy structure of collagen, it is regarded as not supporting compressive stresses. Thus, it is assumed that the fibers contribute to the material response in extension, but are excluded in compression, such that each of the corresponding terms in $W_{\text {aniso }}$ is zero when $I_{4}^{(j)}<1 . I_{4}^{(j)}$ is defined as

$$
I_{4}^{(j)}=\mathbf{C}:\left(\mathbf{a}^{(j)} \otimes \mathbf{a}^{(j)}\right)
$$

where $\mathbf{C}$ designates the right Cauchy-Green deformation tensor, and $\mathbf{a}^{(j)}$ represents a unit vector indicating the mean fiber direction. In all the GI studies, it is assumed that the collagen fibers are embedded in the tangential surface of the tissue (no components in the radial direction). Thus, in a cylindrical polar coordinate system, the direction vector is expressed as

$$
\mathbf{a}^{(j)}=\cos \left(\gamma^{(j)}\right) \mathbf{e}_{\theta}+\sin \left(\gamma^{(j)}\right) \mathbf{e}_{z}
$$

with $\mathbf{e}_{\theta}$ and $\mathbf{e}_{z}$ referring to the circumferential and axial directions in a cylindrical polar coordinate system, respectively and $\gamma^{(j)}$ denoting the angle between the $\mathbf{a}^{(j)}$ and $\mathbf{e}_{\theta}$. In 
215 some studies, $\gamma^{(j)}$ is estimated from microscopy imaging while in others it is considered as an unknown material parameter and estimated from fitting against mechanical testing data. In the original model proposed for arterial wall, a two-fiber family model was proposed with the same stiffness properties $\left(k_{1}^{(1)}=k_{1}^{(2)}\right.$ and $\left.k_{2}^{(1)}=k_{2}^{(2)}\right)$ and such that they symmetrically oriented $\left(\gamma^{(2)}=-\gamma^{(1)}\right)$. Yang and colleagues found that this exact model

220 did not provide a good fit for the mucosa-submucosa and muscle layers of the esophagus against planar uniaxial tests [73]. Looking at their mechanical testing data, they postulated that this model was too nonlinear. Accordingly, they formulated two modified versions of $W_{\text {aniso, }}$, which they called modified exponential and bilinear models. They found that their bilinear model provided the best fit for both esophageal layers. In a subsequent study where they considered tubular inflation-extension tests in addition to the planar uniaxial tests, they found that their modified exponential model provided a better fit [74]. Later studies have shown that rather than a modification of the original Holzapfel model, an extension of the model that captures better the collagen fiber distribution in the GI tissues provided a good fit to the tissue's behavior. Overall, multiple fiber family configurations have been tested and validated for GI tissues: One family [28, 77], two symmetrical fiber families similar to the original Holzapfel model [82, two-fiber families along the longitudinal and circumferential directions [81], three-fiber families along longitudinal and two symmetrical directions $[76,78,79$, and four-fiber families along the longitudinal, circumferential and two symmetrical directions [75, 76, 80]. Typically, the set of parameters $k_{1}$ and $k_{2}$ is assumed to be equal for the two symmetrical directions.

Another structure-based model was presented for the first time for GI tissues by Natali and colleagues in their study of the individual layers of the esophagus [23] before being applied to the intact tissue and layers of the large intestine [24, 25] as well as to the intact tissue of the stomach [83. A list of studies that presented a Natali-type model is provided in Table S5. This type of model uses a split between isotropic and anisotropic contribution similar to the Holzapfel-type models formulated in Equation 13. Different formulations are, however, used for both. The model of the isotropic contribution is formulated as a non-linear function of the three invariants of the right Cauchy-Green deformation tensor C. The model for the anisotropic part account for an exponential function of the fourth invariant similar to the Holzapfel-type models but also consider additional components. 
We refer the readers to the corresponding studies for more details.

One study has proposed a structure-based model with a different formulation for the esophagus tissue, referred to as Microcontinuum Mechanics model [84]. The proposed model is an extension of the Saint-Venant model for classical elasticity to large deformation with an additional parameter that describes the local orientation of fibers (thus making it a structure-based model per definition). We refer the readers to the corresponding study for more details [84].

\subsubsection{Microstructure-based models}

Microstructure-based models account for the microstructural contribution to the macroscopic stress as they incorporate not only the orientation but also the mechanical contribution (strain energy function) of individual fibers. Our search only provided one study investigating a microstructure-based constitutive model for GI tissue. In that study, Puertolas and colleagues [80] conducted planar biaxial testing on intact large intestinal tissue and investigated five constitutive models to capture the passive stress-stain behavior of the tissue: Three Holzapfel-type models (with two symmetrical fiber families, two symmetrical fiber families with consideration of fiber distribution like Gasser [71, fourfiber families with longitudinal, circumferential, and two symmetrical directions) and two microstructure-based models. The two microstructural models proposed were based on the formulation proposed by Alastrué and colleagues for arterial tissue [85, 86. Overall, the models use a split between isotropic and anisotropic contributions as formulated in Eq. (13). The neo-Hookean model was considered for the isotropic part, which is attributed to the ground matrix. The anisotropic part is attributed to the contribution of the collagen fibers and defined as the sum of the contributions of each collagen family of fibers as:

$$
W_{\text {aniso }}=\sum_{j=1}^{N} W_{f, j}
$$

270 where, $\mathrm{N}$ indicates the number of collagen families. Similar to the Holzapfel-type models, a family of fiber is defined as all collagen fibers aligned along a preferred direction. A dispersion of the fibers is, however, considered around this preferred direction as discussed below. For each family $j$, the micro-sphere approach was used to express the overall strain 
energy function $W_{f, j}$ as the average of the contribution, over a unit volume, of each fiber of the family. Specifically, integration of the strain energy of individual fibers $w_{f, j}$ over a unit sphere $S$ was considered such that the contribution of fibers dispersed in all direction is accounted as follows:

$$
W_{f, j}=\frac{1}{4 \pi} \int_{S} n \rho_{j}(\mathbf{r}) w_{f, j}(\lambda(\mathbf{r})) d A
$$

where $\mathbf{r}$ expresses the unit vectors associated with the direction along the spherical integration and $\lambda(\mathbf{r})=\sqrt{\mathbf{F r} \cdot \mathbf{F r}}$ indicates the stretch of the fibers in that direction. Here, $n$ is a constant representing the isotropic network chain density [87], $\rho_{j}$ is the orientation density function (ODF) to take into account the fiber dispersion. A numerical Gaussian quadrature was used to estimate the integral leading to the following expression of $W_{f, j}$ :

$$
W_{f, j}=\sum_{i=1}^{m} \omega_{i} n \rho_{j}\left(\mathbf{r}_{i}\right) w_{f, j}\left(\lambda\left(\mathbf{r}_{i}\right)\right)
$$

where $m$ represents the total number of Gaussian points, and $\left(\omega_{i}, \mathbf{r}_{i}\right)$ the associated sets of Gaussian weights and points. This led to the following overall formulation of $W_{\text {aniso }}$ 285 as:

$$
W_{\text {aniso }}=\sum_{j=1}^{N} \sum_{i=1}^{m} \omega_{i} n \rho_{j}\left(\mathbf{r}_{i}\right) w_{f, j}\left(\lambda\left(\mathbf{r}_{i}\right)\right)
$$

A Holzapfel-type model was proposed for $w_{f, j}$

$$
w_{f, j}(\lambda)=\left\{\begin{array}{c}
\frac{k_{1}^{(j)}}{k_{2}^{(j)}}\left(e^{k_{2}^{(j)}\left(\lambda^{2}-1\right)^{2}}-1\right) \quad \text { if } \quad \lambda \geq 1 \\
0 \quad \text { otherwise }
\end{array}\right.
$$

accounting for the tension-compression switch formulation (i.e., fibers do not support compressive loading). In this study, $N=2$ was chosen based on structural observation of the tissue. However, only one set of parameters $\left(k_{1}, k_{2}, \theta\right)$ was considered indicating that the authors assumed a symmetrical orientation of the preferred directions for the two collagen families. Two different ODFs $\rho_{j}$, respectively known as Microfiber von Mises model and Microfiber Bingham model, were used in the two microstructural models that were 
tested in the study. We refer the readers to the corresponding study for the formulation of the ODFs and additional details. Overall, the study found that the Holzapfel-type four-fiber families model provided the best fit to biaxial testing data, even in comparison to the miscrostructure-based models tested. The microstructure-based models still provided a good fit $\left(0.83<R^{2}<0.95\right.$ for model fitting across specimens for the different locations considered in the study along the colon) with fewer parameters ( 5 vs. 8 for Holzapfel-type four-fiber family model).

\subsection{Viscous and visco-hyperelastic models}

Overall, three studies investigating the visco-hyperelastic behavior of GI tissues were identified, as summarized in Table S6. We outline below the formulation utilized in these studies. We refer the readers to the respective papers as well as classical literature on the topic [88, 89] for more details.

In the first study published in 2007, Higa and colleagues [90 proposed a MooneyRivlin incorporated convolution integral model to characterize the stress relaxation in the colon tissue of the following form:

$$
W^{v}=\int_{0}^{t} \sum_{i+j=1}^{n} g(t-s) \frac{d}{d s}\left[c_{10}\left(I_{1}-3\right)+c_{01}\left(I_{2}-3\right)\right] d s
$$

where $t$ is the time measured with respect to a reference time (e.g., beginning of the experiment), the integral variable $s$ represents an infinitesimal time within time 0 to $t$, and the term under the brackets represents the Mooney Rivlin model presented previously in Equation 11. Here, $g$ represents a normalized relaxation function expressed in terms of the generalized Maxwell's model. In this study, $n=2$ Maxwell elements were selected leading to the following expression of $g$ as:

$$
g(t)=1-g_{1}\left(1-e^{-\frac{t}{\tau_{1}}}\right)-g_{2}\left(1-e^{-\frac{t}{\tau_{2}}}\right)
$$

where $g_{1}, g_{2}$ (normalized modulus) and $\tau_{1}, \tau_{2}$ (relaxation times) are material parameters. The model parameters were estimated from in vivo compression tests at different velocity and resulting model-estimated stress values provided good agreement with experimental data.

Fontanella and colleagues [83] modeled the visco-hyperelastic behavior of the stomach. A Natali-type model, presented in section 5.1.2, was used for the hyperelastic component. 
A viscous variable-based model was used to characterize stress relaxation in the tissue as:

$$
W^{v}=-\sum_{i=1}^{n} \int_{0}^{t} \frac{1}{2} \boldsymbol{q}^{i}(s): \dot{\boldsymbol{C}} d s
$$

This model was previously utilized to characterize the viscous behavior of periodontal ligament [91, 92] and heel pad region [93], among others. Here, $\dot{\boldsymbol{C}}=\frac{\partial \boldsymbol{C}}{\partial t}$ designates the time derivative of the right Cauchy-Green deformation tensor $\boldsymbol{C}, t$ is the time measured with respect to a reference time (e.g., beginning of the experiment), and the integral variable $s$ represents an infinitesimal time within time 0 to $t$. The parameters $n$ indicate the number of viscous elements in the model $[88,89]$. It was assumed in this study that $n=2$. The evolution of the viscous variables $\boldsymbol{q}$ is determine by the following differential equation

$$
\dot{\boldsymbol{q}}^{i}+\frac{1}{\tau^{i}} \boldsymbol{q}^{i}=\frac{\gamma^{i}}{\tau^{i}} 2 \mathbf{F} \frac{\partial W^{e}(\mathbf{C}(t))}{\mathbf{C}}
$$

where $\gamma^{i}$ and $\tau_{i}(i=1,2)$ are material parameters of the model. The parameters of the hyperelastic model were estimated using previously published planar uniaxial tests 94 . Viscous parameters were identified from relaxation curves obtained from ex vivo tubular inflation tests at various inflation/deflation rates and relaxation times. The model was subsequently implemented into a FEM framework and model parameters were further fine tuned to validate the predictions of the FE model against the tubular inflation data.

Panda and Buist [95] investigated the visco-hyperelastic behavior of the small intestine, large intestine, and rectum within a framework proposed previously as an extension of the original work of Huber and colleagues [96, 97. A multiplicative decomposition of the deformation gradient was assumed as:

$$
\mathbf{F}=\mathbf{F}_{e} \mathbf{F}_{i}
$$

where subscripts $e$ and $i$ indicate elastic and inelastic parts of $\boldsymbol{F}$. The following decomposition of the strain energy function was also introduced as:

$$
\Psi=\Psi_{E}(\mathbf{C})+\Psi_{O E}\left(\mathbf{C}_{e}\right)
$$

315 where $\mathbf{C}_{e}=\mathbf{F}_{e}^{T} \mathbf{F}_{e}$, and $\Psi_{E}$ and $\Psi_{O E}$ are strain energy functions associated with the elastic and overstress parts of the deformation, respectively. The full development of the stress-strain relation requires a viscosity model. The authors considered a linear 
viscosity model with a material parameter $\nu$ and a non-linear viscosity model with two material parameters $\mu$ and $\epsilon$. The author applied their model to reproduce several GI mechanical constitutive laws can be formulated requiring the introduction of advanced 
notions of thermodynamics of continuum media [102] enforcing the need for homogenization techniques within a multiphysics framework [103, 104].

A list of studies presenting an active structure-based model is provided in Table S7. We review common formulations of GI tissues' active models according to their historical appearance and mathematical complexity.

\subsection{Active Stress}

The active stress model, initially developed for the cardiac tissue [105, has been typically adopted as a first modeling approximation of the complex excitation-contraction coupling typical of smooth muscle cells, with particular reference to the esophagus [30, 106. 107. An example can be found for the stomach [27] and the small intestine [108, while no publications were found for the large intestine and the rectum using an active stress formulation. As discussed in the literature [109 111, the active stress approach suffers from a rigorous thermodynamical derivation. Accordingly, advanced approaches are briefly discussed in Sec. 6.2

The basic idea of the active stress approach is to superimpose an active contribution $\sigma_{a}$ to the passive (elastic) stress $\boldsymbol{\sigma}_{p}$ such that the total equilibrium Cauchy stress can be defined as:

$$
\sigma=\sigma_{p}+\sigma_{a}
$$

with the pulled-back counterpart expressed in terms of the first Piola-Kirchhoff stress tensor:

$$
\mathbf{P}=\mathbf{P}_{p}+\mathbf{P}_{a}
$$

In such a way, the constitutive modeling can be framed into an invariant reference system and can be used both in small and large strains frameworks. Accordingly, the passive stress can be described by one of the hyperelastic laws described before, whereas the active part is directly coupled with the electrophysiological variable responsible for tissue contraction, e.g., the membrane voltage dynamics (see next section and section S1). Although the electromechanical coupling is due to complex multiscale intracellular calcium dynamics (known as Calcium-induced, Calcium-released or CICR mechanism), the active stress approach usually adopts a phenomenological volumetric formulation where:

$$
\boldsymbol{\sigma}_{a}=T_{a} \mathbf{I}
$$


with

$$
\frac{\mathrm{d} T_{a}}{\mathrm{~d} t}=\epsilon(V)\left(k_{T_{a}} V-T_{a}\right), \quad \epsilon(V)= \begin{cases}\epsilon_{0} & \text { if } \quad V<V_{\mathrm{thr}} \\ N \epsilon_{0} & \text { if } \quad V \geq V_{\mathrm{thr}}\end{cases}
$$

Here, the switch function $\epsilon(V)$ together with the material parameter $k_{T_{a}}$ allows controlling the maximum contractility induced on the tissue according to the level of the membrane voltage $V$. Smooth continuous variants of the switch function $\epsilon(V)$ can also be used to modulate the timing of the contraction.

\subsection{Active Strain}

The active strain approach, originally developed for the cardiac tissue $[109,112$ 115], has been recently applied to the esophagus [82], the stomach [116, and the colon [117] in order to enforce active contractility guided by ICC electrophysiology (see next section and section S1). Adopting the notion of distortion (active deformations [109, 118]) in continuum mechanics, the approach is based on the multiplicative decomposition of the deformation gradient (similar to well-known plasticity theories [119]) into a passive elastic, $\mathbf{F}_{e}$, and an active, $\mathbf{F}_{a}$, part, as:

$$
\mathbf{F}=\mathbf{F}_{e} \mathbf{F}_{a}
$$

In this way, it is possible to enforce multiscale and multiphysics couplings in a continuum homogenized framework through the local active strain deformation map. In particular, intracellular calcium and transmembrane voltage dynamics occur at the cell or subcellular level in much smaller space and time scales with respect to the tissue or organ levels. Accordingly, the local traction-free configuration of the continuum body is herein constitutively related to smooth muscle contractility in terms of infinitesimal volume elements. A representative scheme of such a mapping is provided in Fig. 3, in which an intermediate non-compatible configuration arises due to the presence of the active map ${ }_{375} \mathbf{F}_{a}$.

From a computational point of view, once the active map is known because of muscle contractility, one can compute the elastic deformation gradient by imposing both the balance of linear momentum and geometric compatibility. Moreover, as usual for soft biological media, the tissue is considered incompressible, i.e., $J=\operatorname{det} \mathbf{F}=1$, and further assuming $J_{a}=\operatorname{det} \mathbf{F}_{a}=1$ it follows that $J_{e}=\operatorname{det} \mathbf{F}_{e}=1$. Finally, in view of obtaining 
frame invariant constitutive formulations, the right Cauchy-Green deformation tensors can be introduced from (34) as:

$$
\mathbf{C}=\mathbf{F}^{\mathrm{T}} \mathbf{F}, \quad \mathbf{C}_{e}=\mathbf{F}_{e}{ }^{\mathrm{T}} \mathbf{F}_{e}=\mathbf{F}_{a}{ }^{-\mathrm{T}} \mathbf{C} \mathbf{F}_{a}{ }^{-1}
$$

and the total first and second Piola-Kirchhoff equilibrium stress tensors read, respectively; as:

$$
\mathbf{P}=\frac{\partial \Psi_{e}}{\partial \mathbf{F}_{e}} \frac{\partial \mathbf{F}_{e}}{\partial \mathbf{F}}=\mathbf{P}_{e} \mathbf{F}_{a}^{-\mathrm{T}}, \quad \mathbf{S}=\mathbf{F}^{-1} \mathbf{P}=\mathbf{F}_{a}{ }^{-1} \mathbf{S}_{e} \mathbf{F}_{a}{ }^{-\mathrm{T}}
$$

In the previous equation, $\mathbf{P}_{e}, \mathbf{S}_{e}$ represents the classical elastic first and second PiolaKirchhoff stress tensors, respectively.

The active strain approach constitutive law is completed by the introduction of the specific formulation for the active map $\mathbf{F}_{a}$. As detailed in [116], considering longitudinal and circumferential principal directions of GI muscle contractility, we can write:

$$
\mathbf{F}_{a}=\mathbf{I}-\gamma(V)\left(\alpha_{c} \mathbf{N}_{c} \otimes \mathbf{N}_{c}+\alpha_{l} \mathbf{N}_{l} \otimes \mathbf{N}_{l}\right)+\gamma_{n} \mathbf{N}_{n} \otimes \mathbf{N}_{n}
$$

where $\mathbf{N}_{c}, \mathbf{N}_{l}$ are the orthogonal unit vectors in the circumferential and fiber direction, respectively, defined in the reference configuration and within the plane encompassing SMCs, while $\mathbf{N}_{n}=\mathbf{N}_{c} \times \mathbf{N}_{l}$ represents the orthogonal to the plane thickness direction. In Equation (37), $\alpha_{c}, \alpha_{l}$ represent material parameters ruling the amount of contractility in the corresponding directions 1 , while $\gamma_{n}$ enforces incompressibility as:

$$
\gamma_{n}=\frac{1-\left(1-\gamma \alpha_{c}\right)\left(1-\gamma \alpha_{l}\right)}{\left(1-\gamma \alpha_{c}\right)\left(1-\gamma \alpha_{l}\right)}
$$

Finally, the excitation function $\gamma(V)$ couples the mechanical problem with the electrophysiological one (see next section), in particular via a smooth activation function defined in [116] in terms of the transmembrane voltage of SM as:

$$
\gamma(V)=\left(1-e^{1-\beta_{1}\left(V-V_{\mathrm{thr}}\right)}\right)\left(1-e^{1-\beta_{2}\left(V-V_{\mathrm{thr}}\right)}\right) H\left(V-V_{\mathrm{thr}}\right)
$$

where $\beta_{1}, \beta_{2}, V_{\mathrm{thr}}$ are material parameters linked to the intracellular $\mathrm{Ca} 2+$ dynamics, while $H\left(V-V_{\text {thr }}\right)$ is a Heaviside step function switching on the active contraction whenever the threshold $V_{\text {thr }}$ is reached.

\footnotetext{
${ }^{1}$ Note that (37) can be recast also in the usual structural tensor formalism.
} 


\subsection{Active Electromechanics}

Active stress and active strain approaches have been recently collected under a sound thermodynamical framework [120] generalizing the constitutive modeling of active media electromechanics in the case of the small intestine [121, 122]. To this end, Maxwell electrostatics theory has been introduced, dealing with the material electric field $\mathbf{E}=$ $-\nabla_{\mathbf{X}} V$ defined as the gradient of the electric potential (i.e., the transmembrane voltage $V$ ), the material electric induction $\mathbf{D}$, and the polarization tensors $\boldsymbol{\Pi}$ linked through the following constitutive relation:

$$
\mathbf{D}=J \epsilon_{0} \mathbf{C}^{-1} \mathbf{E}+\mathbf{\Pi}, \quad \nabla_{\mathbf{X}} \cdot \mathbf{D}=\mathbf{0}
$$

where $\epsilon_{0}$ is the vacuum dielectric constant and $\boldsymbol{\Pi}$ accounts for electric distorsions due to materials deformations. Following previous works [120, 123 125], the local thermodynamics state is defined through an electrical Helmholtz free energy $A$ characterized by the functional dependence on $\mathbf{F}, \mathbf{E}$ and a collection of internal variables $\mathbf{Q}$, such that:

$$
\mathbf{P}=\frac{\partial A}{\partial \mathbf{F}}, \quad \mathbf{D}=-\frac{\partial A}{\partial \mathbf{E}}, \quad \mathbf{Y}=-\frac{\partial A}{\partial \mathbf{Q}}
$$

where $\mathbf{P}$ is the equilibrium stress and $\mathbf{Y}$ the thermodynamic forces work-conjugate to Q. According to classical theories of multiphysics couplings in finite elasticity, an additive decomposition of the free energy is assumed in conjunction with the multiplicative decomposition of $\mathbf{F}$ (see Eq. (34)), such that:

$$
A\left(\mathbf{F}, \mathbf{F}_{e}, \mathbf{E}\right)=A_{e}\left(\mathbf{F}_{e}\right)+A_{a}(\mathbf{F}, \mathbf{E})
$$

and the associated stress assumes an additive composition similar to the active stress approach, (31) and 36. In other words, $\mathbf{P}_{a}$ assumes an explicit expression when a constitutive prescription of $\mathbf{F}_{a}$ is provided together with the active inelastic potential.

In this regard, the directional active Helmholtz free energy can be decomposed into isotropic and anisotropic contributions of the GI wall based on the functional dependencies on the electric field $\mathbf{E}$, the fiber direction $\mathbf{n}$, and associated structure tensor $\mathbf{G}=\mathbf{n} \otimes \mathbf{n}$ (by means of the fourth invariant $I_{4}(\mathbf{n})=\mathbf{C}: \mathbf{G}$ ) as:

$$
A_{a}(\mathbf{E}, \mathbf{n})=A_{a}^{\text {iso }}(\mathbf{E})+A_{a}^{\text {aniso }}(\mathbf{E}, \mathbf{n})
$$


385 (see original paper [120]), the active energy formulations state:

$$
\begin{aligned}
A_{a}^{\text {iso }}(\mathbf{E}) & =-\frac{1}{2} \epsilon_{0} J \mathbf{E} \cdot\left[\mathbf{C}^{-1}+\chi_{\text {iso }}\left(I_{1}\right)\right] \mathbf{E} \\
A_{a}^{\text {aniso }}(\mathbf{E}, \mathbf{n}) & =-\frac{1}{2} \epsilon_{0} J \mathbf{E} \cdot \chi_{\text {aniso }}\left(I_{4}(\mathbf{n})\right) \mathbf{E}
\end{aligned}
$$

with $\chi_{\text {iso }}, \chi_{\text {aniso }}$ the associated permittivity tensors. Accordingly, the final expression of the active stress, in terms of the second Piola-Kirchhoff stress tensor, is:

$$
\mathbf{S}_{a}=\mathbf{S}_{a}^{\mathrm{iso}}+\mathbf{S}_{a}^{\text {aniso }}
$$

Visco-electro-elastic distributed models. In Refs. [122, 124, 125], the active electromechanics setting was further extended to include viscous deformation and stochastic material properties [126, 127] to model the colon wall. Assuming a generalized multiplicative decomposition of the deformation gradient (see Fig. 3):

$$
\mathbf{F}=\mathbf{F}_{e}(t) \mathbf{F}_{v}(t) \mathbf{F}_{a}, \quad \mathbf{F}_{e}(t)=\mathbf{F} \mathbf{F}_{a}^{-1} \mathbf{F}_{v}^{-1}
$$

the viscous deformation can be considered as an internal variable, $\mathbf{F}_{v}=\mathbf{Q}$, governed by suitable kinetics. Accordingly, the Helmholtz free energy density becomes:

$$
A=A_{e}\left(\mathbf{F}_{e}\right)+A_{a}(\mathbf{F}, \mathbf{E})+A_{v}\left(\mathbf{F}_{v}, \mathbf{E}, \Delta t\right)
$$

from which the total first Piola-Kirchhoff stress tensor reads:

$$
\mathbf{P}=\mathbf{P}_{\mathrm{E}}+\mathbf{P}_{\mathrm{v}}
$$

where $\mathbf{P}_{\mathrm{E}}=\mathbf{P}_{p}+\mathbf{P}_{a}$ represents the equilibrium stress, e.g., from (41), and the constitutive prescription of the viscous stress is provided by a suitable pseudo potential $\Psi^{*}$ as:

$$
\mathbf{P}_{\mathrm{v}}=\frac{\partial \Psi^{*}}{\partial \dot{\mathbf{F}}}
$$

Furthermore, the active deformation gradient, $\mathbf{F}_{a}$, incorporates directional-dependent stochastic material properties, as key features of GI wall, by means of the functional dependence on a distributed angular variable $\beta$, i.e.:

$$
\mathbf{F}_{a}=f_{1}(\mathbf{E}) \mathbf{I}+f_{2}(\mathbf{E}) \cos \beta^{2} \mathbf{G}
$$


Here, $\beta$ refers to the angle between the aleatoric fiber direction, a, and the electric field unit vector $\mathbf{m}$ :

$$
\cos \beta=\mathbf{m} \cdot \mathbf{a}, \quad \mathbf{m}=\frac{\mathbf{E}}{|\mathbf{E}|}
$$

such that the expected value (integral average) of the active deformation gradient becomes:

$$
\mathbf{F}_{a}=f_{1}(\mathbf{E}) \mathbf{I}+f_{2}(\mathbf{E}) \int_{\Omega} \cos \beta^{2} \mathbf{G} \rho(\mathbf{a}) d \omega=f_{1}(\mathbf{E}) \mathbf{I}+f_{2}(\mathbf{E}) \mathbf{M}: \mathcal{H}
$$

according to the usual structural tensor approach. Here, $\mathcal{H}$ is the average fourth order structural tensor associated with the second order approximation worked out for the passive contribution $[126,128,129$ :

$$
\mathbf{M}=\mathbf{m} \otimes \mathbf{m}, \quad \cos \beta^{2} \mathbf{G}=\mathbf{M}: \mathcal{G}, \quad \int_{\Omega} \mathcal{G} \rho(\mathbf{a}) d \omega=\mathcal{H}
$$

\subsubsection{Multiphysics mathematical modeling of GI tissues}

Few attempts have been made to study multiphysics couplings in GI tissue. An excellent example is provided in $[117,[130]$, modeling the thermal coupling within the intestine tissue because of its critical role in paralytic ileus disease. Adopting the approach proposed by Bini et al. [131, intestinal excitability was coupled to the thermal transport problem by using the Pennes bio-heat equation. Accounting for the ability of tissues to remove heat both by passive conduction (diffusion) and blood perfusion, these contributions were combined in a generalized reaction-diffusion PDE of the form:

$$
\underbrace{C_{t} \frac{\partial T}{\partial t}-\nabla \cdot \mathbf{K}(\boldsymbol{x}) \nabla T}_{\text {Diffusive evolution }}-\underbrace{\omega_{m}(T) C_{b}\left(T_{0}-T\right)}_{\text {Blood perfusion }}-\underbrace{q_{m}(T)}_{\text {Metabolic heat }}-\underbrace{p(\boldsymbol{x}, t)}_{\text {External stimulation }}=0
$$

We refer to the original work [130] for details on model formulation and implementation. Of note, this work was the first to recognize and theoretically anticipate the existence of spiral waves in the intestine (dysrhythmias), which were later experimentally demonstrated 132 136].

The role of ion channels mechanosensitivity [137] and recent advances in the electrophysiological mathematical modeling of GI is referred to recent reviews on the subject [101]. 


\section{Cross-analysis of the reviewed studies}

We present a cross-analysis to provide an overview of the existing studies for modeling GI tissue mechanics and to help identify remaining gaps.

\subsection{Overview}

A total of 54 studies were found to fit our review criteria (when counting individuinvestigated a visco-hyperelastic model, and 9 (17\%) investigated an active model (with or without passive or viscous behavior). The majority of all the studies (56\%) were on esophagus and large intestine tissues. Most of the active studies (44\%) were on the esophagus while active modeling of the rectal tissue has not been investigated to date. These findings are presented in Figures 4.

Looking at the chronological distribution of the studies, shown in Figure 5 , we observe that the first study ever published was in 1994 for the esophagus and introduced a constitutive model for the hyperelastic behavior. No studies were subsequently published for almost a decade, until 2003. Since then, studies have regularly been published, with the first study on the small intestine, large intestine, stomach, and rectum published respectively in 2003, 2007, 2008, and 2009. The first study investigating a visco-hyperelastic model was published in 2007 and the first study investigating an active model was published that same year.

\subsection{Species}

Tissue source for mechanical testing was clearly identifiable in 50 studies. Seven different types of species were used for the tissue source across them (Figure 6). Swine $(45 \%)$ and rodent $(29 \%)$ were the most commonly used while human tissues were used in 5 studies $(11 \%)$.

Among those studies, 6 studies investigated the effect of a disease on the mechanical behavior of GI tissue. Five of the studies were on the small intestine and investigated the hyperelastic behavior of the tissue in diabetic rats [37], active behavior in patients with systemic sclerosis [108], hyperelastic behavior in obstructed guinea pigs [42], hyperelastic behavior in obstructed rats [47], and visco-hyperelastic behavior in patients 
with Hirschsprung's disease [97]. One study investigated the hyperelastic behavior of the

425

\subsection{Mechanical testing condition}

The mechanical testing condition and protocol used to conduct the testing necessary for estimating the material parameters of the constitutive models and/or validating the models were identifiable in 50 studies (7). In 48 studies (96\%), the model parameters were estimated based on testing conducted in ex vivo conditions. A total of 14 different protocols were identified. Planar uniaxial testing (26\%) and tubular inflation-extension testing $(22 \%)$ were most commonly utilized. The two in vivo studies consisted of compression testing in the large intestine of goat [90] and impedance planimetry in the small intestine of humans [108].

\subsection{Model types}

Most of the 40 studies on hyperelastic model considered a phenomenological model (56\%), followed by a structure-based model (42\%). Only one study recently (2019) established a microstructure-based model [80]. Fung-type models (35\%) and Holzapfel-type models (31\%) were the most commonly used (Figure 8).

Limited number of studies (5) investigated the visco-hyperelastic behavior of GI tissues as discussed in section 5.2.

Overall, most of the 9 studies where an active model is established considered active stress model (56\%), followed by an active strain model (33\%) (Figure 8). Only one study established an active electromechanics model that considered a strain-energy function for modeling the active response [127].

\subsection{Studies on human tissues}

An overview of the studies proposing a model based on human tissue testing is provided in Table S8. A total of six such studies have been identified in our review. They proposed models for the intact small intestine [95, 108], large intestine [63], and rectum tissue $[62,67]$ as well as the muscle layer of the large intestine [117]. All but one study on the small intestine [95] were based on ex vivo testing. Three of the studies focused on hyperelastic modeling and all proposed phenomenological models [62, 63, 67], one focused on visco-hyperelastic modeling [95], and two focused on active modeling [66, 117]. 


\section{Discussions}

455 8.1. Overview of the current effort

While the development of constitutive models for biological tissues started as early as the 70s with the advent of engineering approaches in the study of organs and tissues, the gastroenterology field was somehow sidetracked from this revolution. It started to draw serious attention only in the early 2000s. The first study on the constitutive modeling of

460 GI tissues was published in 1994 but consistent attention to the topic has only been drawn from 2003 and the first studies investigating visco-hyperelastic and active tissue models only appeared in 2007. This is a relatively late start compared to the cardiovascular area where studies on the passive and active constitutive modeling for arterial tissues have been initiated in the early 70's and late 80's, respectively. It is thus not surprising that constitutive models of GI tissues are highly inspired by cardiovascular tissue.

To date, there is a disparity of effort attributed to the different GI organs. The stomach and the rectum have been especially neglected despite the prevalent disorders affecting them and the known role/consequence of these conditions on the mechanical behavior of these organs. No study has investigated the behavior of the stomach, large intestine,

470 or rectum under disease conditions. Similarly, the majority of studies have focused on hyperelastic models while limited effort has been dedicated to visco-hyperelastic and active tissue modeling.

\subsection{Recommendations for using existing models}

Our review has identified a number of studies that can be readily used to predict

the stress-behavior of GI tissues. When simple geometry and boundary conditions are of interest, these could be used directly for estimating stress/strain values analytically. For more complex studies, these models can also be implemented in a numerical framework, using for instance the FEM [27, 31, 67, 69, 138].

Choice of the hyperelastic model requires the most attention since a variety of models have been established for GI tissues. It has been shown that phenomenological models typically fail to predict deformation modes outside of those used for estimating their material parameters [139]. Thus, it is recommended to use phenomenological models only when the simulation of interest is within the range of deformation/load used for 
establishing their material parameters. Especially, all the isotropic hyperelastic models for GI tissues have been established based on uniaxal behavior, suggesting that they are only suitable to predict the behavior of the tissue along uni-directional loadings. Structurebased models have greater predictive power and are thus typically recommended. A structure-based model has been established for all the GI organs. While no hyperelasticspecific study has been identified in our review for the stomach, Klemm and colleagues have proposed one as part of their study on the active modeling of the stomach.

Only three manuscripts have investigated modeling of the visco-hyperelastic behavior of the GI tissues. They studied the stomach, large intestine, and rectum. Given the scarcity of such models, selection of a model is simply guided by availability for the tissue of interest.

Although the critical role of smooth contractile cells into the modeling part is wellestablished, studies on active models development for GI tissues are still very limited. The complexity of the phenomenon and its intrinsic multiscale nature require further effort to produce reliable and robust modeling tools for device design and optimization.

\subsection{Suggestions for future studies}

Microstructure-based hyperelastic models are now commonplace for cardiovascular tissues [140, 141]. To date, only one such model has been established (for the large intestine). It is thus recommended that future hyperelastic studies investigate such models. Visco-hyperelastic models are overall scarce, and especially lacking for the esophagus. Active models have attracted more attention in recent years, but none have been validated for the rectum. In general, advanced experimental techniques are required, based on well-established examples in the cardiac domain, characterizing active and passive contributions independently for the different tract of GI. Moreover, only one study has established a full model of the strain energy function incorporating hyperelastic, viscous, and active components. More of such studies are critically needed to capture accurately the full in vivo behavior of GI tissues.

We found only a few studies investigating the role of GI diseases on the mechanical response of the tissues. Such studies are crucial and must be pursued to expand our knowledge of GI mechanics in diseases and also enable accurate simulations of diseased tissue and prediction of treatment outcomes. They are made possible by animal models 
of GI diseases and disorders that have been established over the years that could be used as the source for diseased tissue 142 145].

Although animal tissue based studies provide very useful knowledge of the overall behavior of a tissue, human tissue based studies are clearly needed to evolve towards in silico patient-specific diagnosis and treatment strategies. Across the study reviewed, only six studies proposed a model of human tissues and only one was based on in vivo measurements (small intestine). The development of technologies enabling in vivo and minimally invasive measurements of GI tissue mechanics is also critical to support such modeling effort [146 149].

\subsection{Closing comments}

${ }_{525}$ Notwithstanding the substantial importance of GI mechanics in our everyday life, the research and industry efforts do not compare with the level of modeling, analysis, and investment associated with cardiovascular mechanics. The development of a comprehensive constitutive and computational model of GI mechanics, parametrized for each different section, can lead to patient-specific in-silico tools unveiling the principles governing food digestion and disease mechanisms at different scales. Therefore, such a paradigm stands as an unprecedented opportunity for computer-aided medicine, innovative device design, and optimal treatment approaches. Although modeling approaches can be adapted from the cardiovascular field, GI-specific technologies are needed to gather mechanical behavior data needed to inform and validate such models, especially in humans.

\section{Acknowledgement}

We thank funding from NIH SPARC OT2OD025308 and OT2OD028203.

\section{Author contributions}

All authors have participated sufficiently in the work. B. Patel and A. Gizzi contributed to conception and design of the study, acquisition/analysis/interpretation of data, and drafing/revising the manuscript. H. Gregersen and G.S. Kassab contributed to concept and design of the study, interpretation of data, and revising the manuscript. 


\section{Competing interest statement}

The authors declare no competing interests.

\section{Appendix A. Notation}

We denote $\Omega, \Omega_{s} \in \mathbb{R}$ a deformable body in the reference, spatial configuration with smooth boundary $\partial \Omega, \partial \Omega_{s}$ and outward unit normal $\mathbf{N}, \mathbf{n}$, respectively. A material point in $\Omega, \Omega_{s}$ is denoted by $\mathbf{X}, \mathbf{x}$, respectively, with motion $\mathbf{x}=\mathbf{x}(\mathbf{X}, t)$. Material and spatial gradient, divergence, and determinant operators are identified with $\nabla_{\mathbf{X}}, \nabla_{\mathbf{x}}, \nabla_{\mathbf{X}} \cdot, \nabla_{\mathbf{x}}$, and $\operatorname{det}_{\mathbf{X}}, \operatorname{det}_{\mathbf{x}}$, respectively. Cross, vector, and tensor products are denoted $\cdot, \times, \otimes$. If not explicitly stated, we denote second order tensors in matrix notation with capital bold symbols, $\mathbf{A}$, and corresponding index notation, $A_{I J}$, in reference configuration. Fourth order tensors are indicated with $\mathcal{A}$. Transpose and inverse of a second order tensor are indicated as $\mathbf{A}^{\mathrm{T}}, \mathbf{A}^{-1}$, respectively. Single and double tensor contractions are indicated with $\mathbf{A}^{\mathrm{T}} \mathbf{B}$ and $\mathbf{A}: \mathbf{B}$, respectively.

\section{References}

[1] K. K. Reed, R. Wickham, Review of the gastrointestinal tract: from macro to micro, in: Seminars in oncology nursing, Vol. 25, Elsevier, 2009, pp. 3-14.

[2] C. Stillhart, K. Vučićević, P. Augustijns, A. W. Basit, H. Batchelor, T. R. Flanagan, I. Gesquiere, R. Greupink, D. Keszthelyi, M. Koskinen, et al., Impact of gastrointestinal physiology on drug absorption in special populations - -an ungap review, European Journal of Pharmaceutical Sciences 147 (2020) 105280.

[3] R. S. Sandler, J. E. Everhart, M. Donowitz, E. Adams, K. Cronin, C. Goodman, E. Gemmen, S. Shah, A. Avdic, R. Rubin, The burden of selected digestive diseases in the united states, Gastroenterology 122 (5) (2002) 1500-1511.

[4] P. Oustamanolakis, J. Tack, Dyspepsia: organic versus functional, Journal of clinical gastroenterology 46 (3) (2012) 175-190.

[5] P. A. Myer, A. Mannalithara, G. Singh, G. Singh, P. J. Pasricha, U. Ladabaum, Clinical and economic burden of emergency department visits due to gastrointestinal 
diseases in the united states, American Journal of Gastroenterology 108 (9) (2013) $1496-1507$.

[6] C. Canavan, J. West, T. Card, The economic impact of the irritable bowel syndrome, Alimentary pharmacology \& therapeutics 40 (9) (2014) 1023-1034.

[7] A. J. Gawron, D. D. French, J. E. Pandolfino, C. W. Howden, Economic evaluations of gastroesophageal reflux disease medical management, Pharmacoeconomics 32 (8) (2014) 745-758.

[8] R. K. Wong, D. A. Drossman, Quality of life measures in irritable bowel syndrome, Expert review of gastroenterology \& hepatology 4 (3) (2010) 277-284.

[9] N. Ranasinghe, N. M. Devanarayana, S. Rajindrajith, M. S. Perera, S. Nishanthinie, T. Warnakulasuriya, P. T. de Zoysa, Functional gastrointestinal diseases and psychological maladjustment, personality traits and quality of life, BMC gastroenterology 18 (1) (2018) 1-10.

[10] A. D. Sperber, S. I. Bangdiwala, D. A. Drossman, U. C. Ghoshal, M. Simren, J. Tack, W. E. Whitehead, D. L. Dumitrascu, X. Fang, S. Fukudo, et al., Worldwide prevalence and burden of functional gastrointestinal disorders, results of rome foundation global study, Gastroenterology 160 (1) (2021) 99-114.

[11] H. Gregersen, G. Kassab, Biomechanics of the gastrointestinal tract, Neurogastroenterology \& Motility 8 (4) (1996) 277-297.

[12] R. Aydin, S. Brandstaeter, F. Braeu, M. Steigenberger, R. Marcus, K. Nikolaou, M. Notohamiprodjo, C. Cyron, Experimental characterization of the biaxial mechanical properties of porcine gastric tissue, Journal of the mechanical behavior of biomedical materials 74 (2017) 499-506.

[13] H. Gregersen, Biomechanics of the gastrointestinal tract: new perspectives in motility research and diagnostics, Springer-Verlag London, 2003.

[14] M. Marino, Constitutive modeling of soft tissues, in: R. Narayan (Ed.), Encyclopedia of Biomedical Engineering, Elsevier, 2019, pp. 81-110. 
¿ [15] B. Patel, Dataset v1.0.0: Biomechanical constitutive modeling of the gastrointestinal tissues (2021).

URL http: //dx .doi .org/10.5281/zenodo.5495996

[16] M. D. Wilkinson, M. Dumontier, I. J. Aalbersberg, G. Appleton, M. Axton,

A. Baak, N. Blomberg, J.-W. Boiten, L. B. da Silva Santos, P. E. Bourne, et al., The fair guiding principles for scientific data management and stewardship, Scientific data 3 (1) (2016) 1-9.

[17] B. Patel, H. Srivastava, P. Aghasafari, K. Helmer, Sparc: Soda, an interactive software for curating sparc datasets, The FASEB Journal 34 (S1) (2020) 1-1.

[18] A. Bandrowski, J. S. Grethe, A. Pilko, T. H. Gillespie, G. Pine, B. Patel, M. SurlesZeiglera, M. E. Martone, Sparc data structure: Rationale and design of a fair standard for biomedical research data, bioRxiv (2021).

[19] M. Osanlouy, A. Bandrowski, B. de Bono, D. Brooks, A. M. Cassarà, R. Christie, N. Ebrahimi, T. Gillespie, J. S. Grethe, L. A. Guercio, et al., The sparc drc: Building a resource for the autonomic nervous system community, bioRxiv (2021).

[20] G. A. Holzapfel, Nonlinear Solid Mechanics: A Continuum Approach for Engineering, John Wiley \& Sons, New York, 2000.

[21] J. D. Humphrey, Continuum biomechanics of soft biological tissues, Proc. R. Soc. Lond. A 459 (2003) 3-46.

[22] R. W. Ogden, Nonlinear continuum mechanics and modeling the elasticity of soft biological tissues with a focus on artery walls, in: Biomechanics: Trends in Modeling and Simulation, Springer, 2017, pp. 83-156.

[23] A. N. Natali, E. L. Carniel, H. Gregersen, Biomechanical behaviour of oesophageal tissues: material and structural configuration, experimental data and constitutive analysis, Medical engineering \& physics 31 (9) (2009) 1056-1062.

[24] E. Carniel, V. Gramigna, C. Fontanella, A. Frigo, C. Stefanini, A. Rubini, A. Natali, Characterization of the anisotropic mechanical behaviour of colonic tissues: 
experimental activity and constitutive formulation, Experimental physiology 99 (5) (2014) 759-771.

[25] E. L. Carniel, V. Gramigna, C. G. Fontanella, C. Stefanini, A. N. Natali, Constitutive formulations for the mechanical investigation of colonic tissues, Journal of biomedical materials research Part A 102 (5) (2014) 1243-1254.

[26] A. Gizzi, A. Pandolfi, M. Vasta, Viscoelectromechanics modeling of intestine wall hyperelasticity, International Journal for Computational Methods in Engineering Science and Mechanics 17 (3) (2016) 143-155.

[27] L. Klemm, R. Seydewitz, M. Borsdorf, T. Siebert, M. Böl, On a coupled electrochemomechanical model of gastric smooth muscle contraction, Acta biomaterialia 109 (2020) 163-181.

[28] Y. Zhao, S. Siri, B. Feng, D. Pierce, Computational modeling of mouse colorectum capturing longitudinal and through-thickness biomechanical heterogeneity, Journal of the Mechanical Behavior of Biomedical Materials 113 (2021) 104127.

[29] R. P. Vito, S. A. Dixon, Blood vessel constitutive models-1995-2002, Annual review of biomedical engineering 5 (1) (2003) 413-439.

[30] R. Yassi, L. Cheng, V. Rajagopal, M. Nash, J. Windsor, A. Pullan, Modeling of the mechanical function of the human gastroesophageal junction using an anatomically realistic three-dimensional model, Journal of biomechanics 42 (11) (2009) 16041609.

[31] S. Dargar, A. C. Akyildiz, S. De, In situ mechanical characterization of multilayer soft tissue using ultrasound imaging, IEEE Transactions on Biomedical Engineering 64 (11) (2016) 2595-2606.

[32] M. Destrade, G. Saccomandi, I. Sgura, Methodical fitting for mathematical models of rubber-like materials, Proceedings of the Royal Society A 473 (2017) 20160811.

[33] A. B. Tepole, D. Nordsletten, K. Garikipati, E. Kuhl, Special issue on uncertainty quantification, machine learning, and data-driven modeling of biological systems, Computer Methods in Applied Mechanics and Engineering 362 (112832) (2020). 
[34] R. Miftakhov, D. Wingate, Biomechanics of small bowel motility, Medical engineering \& physics 16 (5) (1994) 406-415.

[35] D. Liao, Y. Fan, Y. Zeng, H. Gregersen, Stress distribution in the layered wall of the rat oesophagus, Medical engineering \& physics 25 (9) (2003) 731-738.

[36] Y. Dou, J. Zhao, H. Gregersen, Morphology and stress-strain properties along the small intestine in the rat, J. Biomech. Eng. 125 (2) (2003) 266-273.

[37] J. Zhao, J. Yang, H. Gregersen, Biomechanical and morphometric intestinal remodelling during experimental diabetes in rats, Diabetologia 46 (12) (2003) 1688-1697.

[38] Y. Zeng, Y. Qiao, J. Yang, H. Gregersen, E. Zhang, X. Xu, H. Xu, Torque properties of a rat oesophagus for physiological and diabetic conditions, Physiological measurement 25 (5) (2004) 1211.

[39] J. Yang, D. Liao, J. Zhao, H. Gregersen, Shear modulus of elasticity of the esophagus, Annals of biomedical engineering 32 (9) (2004) 1223-1230.

[40] E. A. Stavropoulou, Y. F. Dafalias, D. P. Sokolis, Biomechanical and histological characteristics of passive esophagus: experimental investigation and comparative constitutive modeling, Journal of biomechanics 42 (16) (2009) 2654-2663.

[41] D. P. Sokolis, Strain-energy function and three-dimensional stress distribution in esophageal biomechanics, Journal of biomechanics 43 (14) (2010) 2753-2764.

[42] D. Liao, J. Zhao, H. Gregersen, 3d mechanical properties of the partially obstructed guinea pig small intestine, Journal of biomechanics 43 (11) (2010) 2079-2086.

[43] C. Bellini, P. Glass, M. Sitti, E. S. Di Martino, Biaxial mechanical modeling of the small intestine, Journal of the mechanical behavior of biomedical materials 4 (8) (2011) 1727-1740.

[44] D. P. Sokolis, I. K. Orfanidis, M. Peroulis, Biomechanical testing and material characterization for the rat large intestine: regional dependence of material parameters, Physiological measurement 32 (12) (2011) 1969. 
[45] E. A. Stavropoulou, Y. F. Dafalias, D. P. Sokolis, Biomechanical behavior and histological organization of the three-layered passive esophagus as a function of topography, Proceedings of the Institution of Mechanical Engineers, Part H: Journal of Engineering in Medicine 226 (6) (2012) 477-490.

[46] D. P. Sokolis, Experimental study and biomechanical characterization for the passive small intestine: identification of regional differences, Journal of the mechanical behavior of biomedical materials 74 (2017) 93-105.

[47] D. Sun, J. Zhao, D. Liao, P. Chen, H. Gregersen, Shear modulus of the partially obstructed rat small intestine, Annals of biomedical engineering 45 (4) (2017) 1069 1082.

[48] Y.-c. Fung, N. Perrone, M. Anliker, et al., Biomechanics, its foundations and objectives, in: Symposium on Biomechanics, its Foundations and Objectives (1970: University of California, San Diego), NJ, Prentice-Hall, 1972.

690

[49] Y. Fung, K. Fronek, P. Patitucci, Pseudoelasticity of arteries and the choice of its mathematical expression, American Journal of Physiology-Heart and Circulatory Physiology 237 (5) (1979) H620-H631.

[50] C. J. Chuong, Y. C. Fung, Three-Dimensional Stress Distribution in Arteries, Journal of Biomechanical Engineering 105 (3) (1983) 268-274. URL https://doi .org/10.1115/1.3138417

[51] S. Deng, J. Tomioka, J. Debes, Y. Fung, New experiments on shear modulus of elasticity of arteries, American Journal of Physiology-Heart and Circulatory Physiology 266 (1) (1994) H1-H10.

[52] J. D. Humphrey, Mechanics of the arterial wall: review and directions, Critical Reviews $^{\mathrm{TM}}$ in Biomedical Engineering 23 (1-2) (1995).

[53] W. W. von Maltzahn, R. G. Warriyar, W. F. Keitzer, Experimental measurements of elastic properties of media and adventitia of bovine carotid arteries, Journal of biomechanics 17 (11) (1984) 839-847. 
[54] F. C. Yin, R. K. Strumpf, P. H. Chew, S. L. Zeger, Quantification of the mechanical properties of noncontracting canine myocardium under simultaneous biaxial loading, Journal of biomechanics 20 (6) (1987) 577-589.

[55] J. Humphrey, R. Strumpf, F. Yin, A constitutive theory for biomembranes: application to epicardial mechanics (1992).

[56] P. Tong, Y.-C. Fung, The stress-strain relationship for the skin, Journal of Biomechanics 9 (10) (1976) 649-657.

[57] H. Demiray, H. Weizsäcker, K. Pascale, A mechanical model for passive behaviour of rats carotid artery. ein mechanisches modell für das passive verhalten der arteria carotis von ratten, Biomedical Engineering/Biomedizinische Technik 31 (3) (1986) $46-52$.

[58] Y. Fung, S. Liu, J. Zhou, Remodeling of the constitutive equation while a blood vessel remodels itself under stress (1993).

[59] J. Humphrey, D. Vawter, R. Vito, Pseudoelasticity of excised visceral pleura., Journal of biomechanical engineering 109 (2) (1987) 115-120.

[60] P. H. Chew, F. C. Yin, S. L. Zeger, Biaxial stress-strain properties of canine pericardium, Journal of molecular and cellular cardiology 18 (6) (1986) 567-578.

[61] S. Brandstaeter, A. Gizzi, S. L. Fuchs, A. M. Gebauer, R. C. Aydin, C. J. Cyron, Computational model of gastric motility with active-strain electromechanics, ZAMM-Journal of Applied Mathematics and Mechanics/Zeitschrift für Angewandte Mathematik und Mechanik 98 (12) (2018) 2177-2197.

[62] C. Rubod, M. Brieu, M. Cosson, G. Rivaux, J.-C. Clay, L. de Landsheere, B. Gabriel, Biomechanical properties of human pelvic organs, Urology 79 (4) (2012) 968-e17.

[63] S. Puértolas, E. Bajador, J. A. Puértolas, E. López, E. Ibarz, L. Gracia, A. Herrera, Study of the behavior of a bell-shaped colonic self-expandable niti stent under peristaltic movements, BioMed research international 2013 (2013). 
[64] D. Zhou, X. He, Numerical evaluation of the efficacy of small-caliber colonoscopes in reducing patient pain during a colonoscopy, Computer methods in biomechanics and biomedical engineering 22 (1) (2019) 38-46.

[65] R. W. Ogden, Large deformation isotropic elasticity-on the correlation of theory and experiment for incompressible rubberlike solids, Proceedings of the Royal Society of London. A. Mathematical and Physical Sciences 326 (1567) (1972) 565-584.

[66] F. Gao, D. Liao, J. Zhao, A. M. Drewes, H. Gregersen, Numerical analysis of pouch filling and emptying after laparoscopic gastric banding surgery, Obesity surgery 18 (3) (2008) 243-250.

[67] M. B. Boubaker, M. Haboussi, J.-F. Ganghoffer, P. Aletti, Finite element simulation of interactions between pelvic organs: predictive model of the prostate motion in the context of radiotherapy, Journal of biomechanics 42 (12) (2009) 1862-1868.

[68] T. N. Tran, V. Novacek, R. Tolba, U. Klinge, F. Turquier, M. Staat, Experimental and computational approach to study colorectal anastomosis. isb2011, proceedings of the xxiii congress of the international society of biomechanics, brussels, belgium, july 3-7, 2011 (2011).

[69] M. B. Boubaker, M. Haboussi, J.-F. Ganghoffer, P. Aletti, Predictive model of the prostate motion in the context of radiotherapy: a biomechanical approach relying on urodynamic data and mechanical testing, Journal of the mechanical behavior of biomedical materials 49 (2015) 30-42.

[70] G. A. Holzapfel, T. C. Gasser, R. W. Ogden, A new constitutive framework for arterial wall mechanics and a comparative study of material models, Journal of elasticity and the physical science of solids 61 (1-3) (2000) 1-48.

[71] T. C. Gasser, R. W. Ogden, G. A. Holzapfel, Hyperelastic modelling of arterial lay755 ers with distributed collagen fibre orientations, Journal of the royal society interface 3 (6) (2006) 15-35.

[72] G. A. Holzapfel, R. W. Ogden, Constitutive modelling of arteries, Proceedings of the Royal Society A: Mathematical, Physical and Engineering Sciences 466 (2118) (2010) 1551-1597. 
760

765

770

[73] W. Yang, T. Fung, K. Chian, C. Chong, Directional, regional, and layer variations of mechanical properties of esophageal tissue and its interpretation using a structurebased constitutive model (2006).

[74] W. Yang, T. Fung, K. Chian, C. Chong, 3d mechanical properties of the layered esophagus: experiment and constitutive model (2006).

[75] P. Ciarletta, P. Dario, F. Tendick, S. Micera, Hyperelastic model of anisotropic fiber reinforcements within intestinal walls for applications in medical robotics, The International Journal of Robotics Research 28 (10) (2009) 1279-1288.

[76] D. P. Sokolis, Structurally-motivated characterization of the passive pseudo-elastic response of esophagus and its layers, Computers in biology and medicine 43 (9) (2013) 1273-1285.

[77] G. Sommer, A. Schriefl, G. Zeindlinger, A. Katzensteiner, H. Ainödhofer, A. Saxena, G. A. Holzapfel, Multiaxial mechanical response and constitutive modeling of esophageal tissues: impact on esophageal tissue engineering, Acta biomaterialia 9 (12) (2013) 9379-9391.

[78] D. P. Sokolis, S. G. Sassani, Microstructure-based constitutive modeling for the large intestine validated by histological observations, Journal of the mechanical behavior of biomedical materials 21 (2013) 149-166.

[79] B. Patel, H. Chen, A. Ahuja, J. F. Krieger, J. Noblet, S. Chambers, G. S. Kassab, Constitutive modeling of the passive inflation-extension behavior of the swine colon, Journal of the mechanical behavior of biomedical materials 77 (2018) 176-186.

[80] S. Puértolas, E. Peña, A. Herrera, E. Ibarz, L. Gracia, A comparative study of hyperelastic constitutive models for colonic tissue fitted to multiaxial experimental testing, Journal of the mechanical behavior of biomedical materials 102 (2020) 103507.

[81] D. P. Sokolis, Variation of passive biomechanical properties of the small intestine along its length: Microstructure-based characterization, Bioengineering 8 (3) (2021) 32 . 
[82] M. Peirlinck, N. Debusschere, F. Iannaccone, P. D. Siersema, B. Verhegghe, P. Segers, M. De Beule, An in silico biomechanical analysis of the stent-esophagus interaction, Biomechanics and modeling in mechanobiology 17 (1) (2018) 111-131.

[83] C. G. Fontanella, C. Salmaso, I. Toniolo, N. de Cesare, A. Rubini, G. M. De Benedictis, E. L. Carniel, Computational models for the mechanical investigation of stomach tissues and structure, Annals of biomedical engineering 47 (5) (2019) 1237-1249.

[84] D. Sanchez-Molina, J. Velazquez-Ameijide, C. Arregui-Dalmases, D. Rodríguez, V. Quintana, M. Shafieian, J. Crandall, A microcontinuum model for mechanical properties of esophageal tissue: experimental methodology and constitutive analysis, Annals of biomedical engineering 42 (1) (2014) 62-72.

[85] V. Alastrué, M. Martinez, M. Doblaré, A. Menzel, Anisotropic micro-sphere-based finite elasticity applied to blood vessel modelling, Journal of the Mechanics and Physics of Solids 57 (1) (2009) 178-203.

[86] V. Alastrué, P. Sáez, M. Martínez, M. Doblaré, On the use of the bingham statistical distribution in microsphere-based constitutive models for arterial tissue, Mechanics Research Communications 37 (8) (2010) 700-706.

[87] C. Miehe, S. Göktepe, F. Lulei, A micro-macro approach to rubber-like materialspart i: the non-affine micro-sphere model of rubber elasticity, Journal of the Mechanics and Physics of Solids 52 (11) (2004) 2617-2660.

[88] J. C. Simo, T. J. Hughes, Computational inelasticity, Vol. 7, Springer Science \& Business Media, 2006.

[89] A. G. Holzapfel, Nonlinear solid mechanics - a continuum approach for engineering (2000).

[90] M. Higa, Y. Luo, T. Okuyama, Y. Shiraishi, H. Liu, T. Yambe, T. Takagi, In vivo measurements and constitutive modeling of colon tissue, in: World Congress on Medical Physics and Biomedical Engineering 2006, Springer, 2007, pp. 3186-3189.

[91] A. Natali, P. Pavan, E. Carniel, C. Dorow, Viscoelastic response of the periodontal 
815

820

825

ligament: an experimental-numerical analysis, Connective Tissue Research 45 (4-5) (2004) 222-230.

[92] A. N. Natali, E. L. Carniel, P. G. Pavan, F. G. Sander, C. Dorow, M. Geiger, A visco-hyperelastic-damage constitutive model for the analysis of the biomechanical response of the periodontal ligament, Journal of biomechanical engineering 130 (3) (2008).

[93] A. Natali, C. Fontanella, E. Carniel, Constitutive formulation and numerical analysis of the heel pad region, Computer methods in biomechanics and biomedical engineering 15 (4) (2012) 401-409.

[94] J. Zhao, D. Liao, P. Chen, P. Kunwald, H. Gregersen, Stomach stress and strain depend on location, direction and the layered structure, Journal of biomechanics 41 (16) (2008) 3441-3447.

[95] S. K. Panda, M. L. Buist, A viscoelastic framework for inflation testing of gastrointestinal tissue, Journal of the mechanical behavior of biomedical materials 103 (2020) 103569.

[96] N. Huber, C. Tsakmakis, Finite deformation viscoelasticity laws, Mechanics of materials 32 (1) (2000) $1-18$.

[97] S. K. Panda, M. L. Buist, A finite nonlinear hyper-viscoelastic model for soft biological tissues, Journal of biomechanics 69 (2018) 121-128.

[98] E. L. Carniel, A. Rubini, A. Frigo, A. N. Natali, Analysis of the biomechanical behaviour of gastrointestinal regions adopting an experimental and computational approach, Computer methods and programs in biomedicine 113 (1) (2014) 338-345.

[99] E. L. Carniel, M. Mencattelli, G. Bonsignori, C. G. Fontanella, A. Frigo, A. Rubini, C. Stefanini, A. N. Natali, Analysis of the structural behaviour of colonic segments by inflation tests: Experimental activity and physio-mechanical model, Proceedings of the Institution of Mechanical Engineers, Part H: Journal of Engineering in Medicine 229 (11) (2015) 794-803. 
[100] P. Arhan, G. Devroede, K. Danis, C. Dornic, C. Faverdin, B. Persoz, D. Pellerin, et al., Viscoelastic properties of the rectal wall in hirschsprung's disease., The Journal of clinical investigation 62 (1) (1978) 82-87.

${ }_{845}^{6}$ [101] P. Du, S. Calder, T. Angeli, S. Sathar, N. Paskaranandavadivel, G. O’Grady, L. Cheng, Progress in mathematical modeling of gastrointestinal slow wave abnormalities, Frontiers in Physiology 8 (2018) 1136.

[102] P. Podio-Guidugli, Continuum thermodynamics (2019).

[103] C. J. Cyron, R. C. Aydin, J. D. Humphrey, A homogenized constrained mixture (and mechanical analog) model for growth and remodeling of soft tissue, Biomechanics and Modeling in Mechanobiology 15 (2016) 1389-1403.

[104] C. J. Cyron, J. D. Humphrey, Growth and remodeling of load-bearing biological soft tissues 52 (2017) 645-664.

[105] P. Hunter, A. McCulloch, H. ter Keurs, Modelling the mechanical properties of cardiac muscle, Progress in Biophysics and Molecular Biology 69 (2) (1998) 289331.

[106] W. Yang, T. Fung, K. Chian, C. Chong, Three-dimensional finite element model of the two-layered oesophagus, including the effects of residual strains and buckling of mucosa, Proceedings of the Institution of Mechanical Engineers, Part H: Journal of Engineering in Medicine 221 (4) (2007) 417-426.

[107] W. Kou, A. P. S. Bhalla, B. E. Griffith, J. E. Pandolfino, P. J. Kahrilas, N. A. Patankar, A fully resolved active musculo-mechanical model for esophageal transport, Journal of Computational Physics 298 (2015) 446-465.

[108] F. Gao, D. Liao, A. M. Drewes, H. Gregersen, Modelling the elastin, collagen and smooth muscle contribution to the duodenal mechanical behaviour in patients with systemic sclerosis, Neurogastroenterology \& Motility 21 (9) (2009) 914-e68.

[109] C. Cherubini, S. Filippi, P. Nardinocchi, L. Teresi, An electromechanical model of cardiac tissue: Constitutive issues and electrophysiological effects, Progress in Biophysics and Molecular Biology 97 (2008) 562-573. 
870 [110] D. Ambrosi, S. Pezzuto, Active stress vs. active strain in mechanobiology: constitutive issues, Journal of Elasticity 107 (2012) 199-212.

[111] G. Giantesio, A. Musesti, D. Riccobelli, A comparison between active strain and active stress in transversely isotropic hyperelastic materials, Journal of Elasticity 137 (2019) 63-82.

${ }_{875}^{6}$ [112] D. Ambrosi, et al., Ambrosi, d and ateshian, gerard a and arruda, ellen $\mathrm{m}$ and cowin, sc and dumais, $\mathrm{j}$ and goriely, a and holzapfel, gerhard a and humphrey, jay d and kemkemer, $\mathrm{r}$ and kuhl, ellen and others, Journal of the Mechanics and Physics of Solids 59 (2011) 863-883.

[113] F. Nobile, A. Quarteroni, R. Ruiz-Baier, An active strain electromechanical model

880

[115] R. Ruiz-Baier, A. Gizzi, S. Rossi, C. Cherubini, A. Laadhari, S. Filippi, A. Quarteroni, Mathematical modelling of active contraction in isolated cardiomyocytes, Mathematical Medicine and Biology 31 (2014) 259-283.

[116] S. Brandstaeter, S. L. Fuchs, R. C. Aydin, C. J. Cyron, Mechanics of the stomach: A review of an emerging field of biomechanics, GAMM - Mitteilungen e201900001 (2019) 1-17.

[117] A. Altomare, A. Gizzi, M. P. Guarino, A. Loppini, S. Cocca, M. Dipaola, R. Alloni, M. Cicala, S. Filippi, Experimental evidence and mathematical modeling of thermal effects on human colonic smooth muscle contractility, American Journal of Physiology-Gastrointestinal and Liver Physiology 307 (1) (2014) G77-G88.

${ }_{895}$ [118] L. Teresi, P. Nardinocchi, On the active response of soft living tissues, Journal of Elasticity 88 (2007) 27-39. 
[119] V. A. Lubarda, Constitutive theories based on the multiplicative decomposition of deformation gradient: Thermoelasticity, elastoplasticity, and biomechanics, Appl. Mech. Rev. 57 (2004) 95-108.

[120] A. Gizzi, C. Cherubini, S. Filippi, A. Pandolfi, Theoretical and numerical modeling of nonlinear electromechanics with applications to biological active media, Communications in Computational Physics 17 (2015) 93-126.

[121] A. Gizzi, A. Pandolfi, M. Vasta, Viscoelectromechanics modeling of intestine wall hyperelasticity, Int. J. Comput. Methods Eng. Sci. Mech. 17 (2016) 143-155.

905

[122] A. Pandolfi, A. Gizzi, M. Vasta, Coupled electro-mechanical models of fiberdistributed active tissues, J. Biomech. 49 (2016) 2436-2444.

[123] Z. Suo, X. Zhao, W. Greene, A nonlinear field theory of deformable dielectrics, Journal of the Mechanics and Physics of Solids 56 (2008) 467-486.

[124] A. Gizzi, A. Pandolfi, Visco-hyperelasticity of electro-active soft tissues, Procedia IUTAM 12 (2015) 162-175.

[125] A. Pandolfi, A. Gizzi, M. Vasta, Visco-electro-elastic models of fiber-distributed active tissues, Meccanica (2017) 1-17.

[126] M. Vasta, A. Gizzi, A. Pandolfi, On three- and two-dimensional fiber distributed models of biological tissues, Prob. Eng. Mech. 37 (2014) 170-179.

915 [127] A. Gizzi, A. Pandolfi, M. Vasta, Statistical characterization of the anisotropic strain energy in soft materials with distributed fibers, Mechanics of Materials 92 (2016) $119-138$.

[128] A. Pandolfi, M. Vasta, Fiber distributed hyperelastic modeling of biological tissues, Mec. Mat. 44 (2012) 151-162.

920

[129] A. Gizzi, M. Vasta, A. Pandolfi, Modeling collagen recruitment in hyperelastic biomaterial models with statistical distribution of the fiber orientation, International Journal of Engineering Science 78 (2014) 48-60. 
[130] A. Gizzi, C. Cherubini, S. Migliori, R. Alloni, R. Portuesi, S. Filippi, On the electrical intestine turbulence induced by temperature changes, Physical Biology 7 (2010) 016011.

[131] D. Bini, C. Cherubini, S. Filippi, A. Gizzi, P. E. Ricci, On spiral waves arising in natural systems, Communications in Computational Physics 8 (2010) 610.

[132] G. O'Grady, J. U. Egbuji, P. Du, W. E. J. P. Lammers, L. K. Cheng, J. A. Windsor, A. J. Pullan, High-resolution spatial analysis of slow wave initiation and conduction in porcine gastric dysrhythmia, Neurogastroenterology \& Motility 23 (2011) e345e355.

[133] W. E. J. P. Lammers, B. Stephen, S. M. Karam, Functional reentry and circus movement arrhythmias in the small intestine of normal and diabetic rats, American Journal of Physiol Gastrointestine Liver Physiology 302 (2012) G684-G689.

[134] W. E. J. P. Lammers, Arrhythmias in the gut, Neurogastroenterology \& Motility 25 (2013) 353-357.

[135] S. Calder, G. O'Grady, L. K. Cheng, P. Du, A theoretical analysis of electrogastrography (egg) signatures associated with gastric dysrhythmias, IEEE Transactions on Biomedical Engineering 64 (2016) 1592-1601.

[136] P. Du, G. O'Grady, L. K. Cheng, A theoretical analysis of anatomical and functional intestinal slow wave re-entry, Journal of Theoretical Biology 425 (2017) 72-79.

[137] A. Beyder, R. Lees-Green, G. Farrugia, Role of ion channel mechanosensitivity in the gut: Mechano-electrical feedback exemplified by stretch-dependence of nav1.5. (2013).

[138] B. Patel, X. Guo, J. Noblet, S. Chambers, H. Gregersen, G. S. Kassab, Computational analysis of mechanical stress in colonic diverticulosis, Scientific reports 10 (1) (2020) 1-12.

[139] Y. Hollander, D. Durban, X. Lu, G. S. Kassab, Y. Lanir, Constitutive modeling of coronary arterial media - comparison of three model classes, Journal of biomechanical engineering 133 (6) (2011). 
[140] H. Chen, G. S. Kassab, Microstructure-based biomechanics of coronary arteries in health and disease, Journal of biomechanics 49 (12) (2016) 2548-2559.

[141] H. Chen, X. Guo, T. Luo, G. S. Kassab, A validated 3d microstructure-based constitutive model of coronary artery adventitia, Journal of Applied Physiology 121 (1) (2016) 333-342.

[142] E. Glover, M. Leland, E. Dick Jr, G. Hubbard, Gastroesophageal reflux disease in baboons (papio sp.): a new animal model, Journal of medical primatology 37 (1) (2008) 18-25.

[143] J. L. Bohl, E. Zakhem, K. N. Bitar, Successful treatment of passive fecal incontinence in an animal model using engineered biosphincters: A 3-month follow-up study, Stem cells translational medicine 6 (9) (2017) 1795-1802.

[144] B. Patel, X. Guo, J. Noblet, S. Chambers, G. S. Kassab, Animal models of diverticulosis: review and recommendations, Digestive diseases and sciences 63 (6) (2018) 1409-1418.

[145] X. Guo, B. Patel, L. Han, H. Al-Dulaimi, W. G. Van Alstine, J. N. Noblet, S. Chambers, G. S. Kassab, Novel swine model of colonic diverticulosis, American Journal of Physiology-Gastrointestinal and Liver Physiology 317 (1) (2019) G51-G56.

[146] H. Gregersen, K. Krogh, D. Liao, Fecobionics: integrating anorectal function measurements, Clinical Gastroenterology and Hepatology 16 (6) (2018) 981-983.

[147] D. Sun, Z. Huang, Z. Zhuang, Z. Ma, L. K. Man, D. Liao, H. Gregersen, Fecobionics: A novel bionics device for studying defecation, Annals of biomedical engineering 47 (2) (2019) 576-589.

[148] H. Gregersen, S.-C. Chen, W. W. Leung, C. Wong, T. Mak, S. Ng, K. Futaba, Novel fecobionics defecatory function testing, Clinical and translational gastroenterology 10 (12) (2019).

[149] H. Gregersen, Y. Wang, X. Guo, F. Field, M. Nelson, W. Combs, M. Wang, G. Kassab, Simulated colonic feces reveals novel contraction patterns, Gastroenterology 160 (3) (2021) 660-662. 


\section{List of Figures}

980 1 Illustration of the GI tract along with specification of the different organs (edited from www.flickr.com/photos/nihgov/25083237542). The number of studies that fitted our review criteria is indicated in parenthesis for each

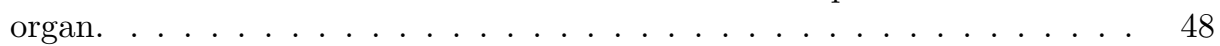

2 PRISMA diagram providing an overview of our review process. . . . . . . 49

985

3 Illustration of the decomposition of the deformation gradient. . . . . . . .

4 Organ-wise distribution of the studies with distinction between studies investigating hyperelastic, visco-hyperelastic, and active models. . . . . . 51

$5 \quad$ Organ-wise and year-wise distribution of the studies with distinction between studies investigating hyperelastic, visco-hyperelastic, and active models. . . . . . . . . . . . . . . . . . . . .

6 Organ-wise distribution of the studies with distinction of the tissue source used for mechanical testing. . . . . . . . . . . . . . . . .

7 Organ-wise distribution of the studies with identification of the mechanical testing protocol utilized to estimate model parameters and/or validate the models. . . . . . . . . . . . . . . . . . . . . . 54

8 Distribution of the studies based on the hyperelastic model type proposed. 55

$9 \quad$ Distribution of the studies based on the active model type proposed. . . . 56




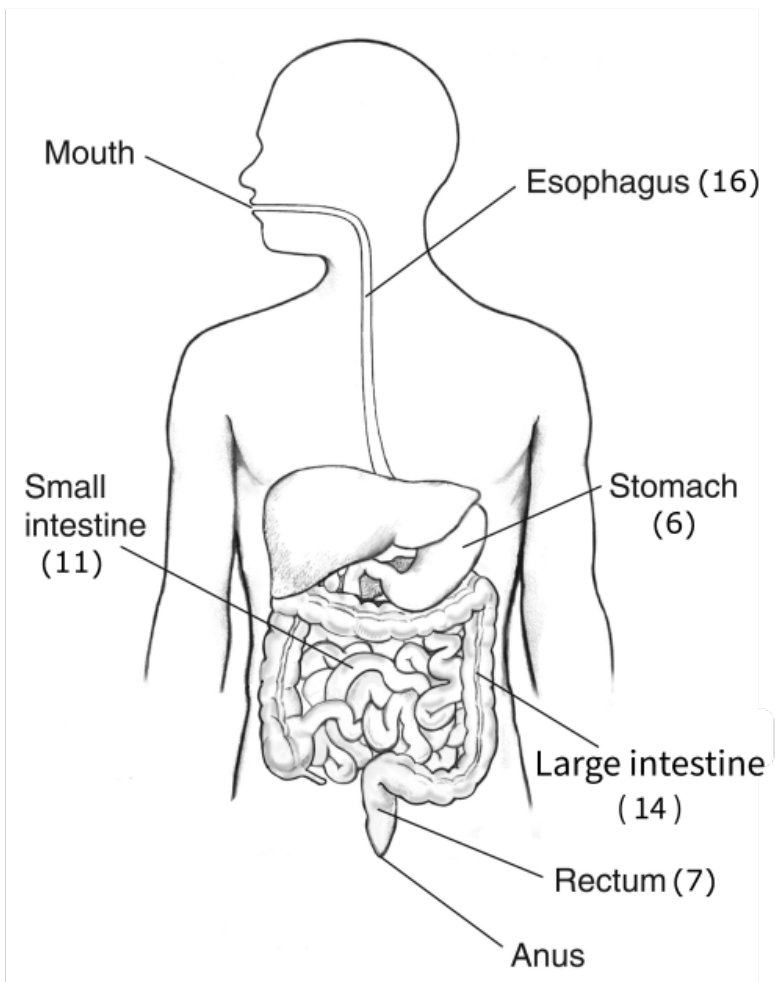

Figure 1: Illustration of the GI tract along with specification of the different organs (edited from www.flickr.com/photos/nihgov/25083237542). The number of studies that fitted our review criteria is indicated in parenthesis for each organ. 


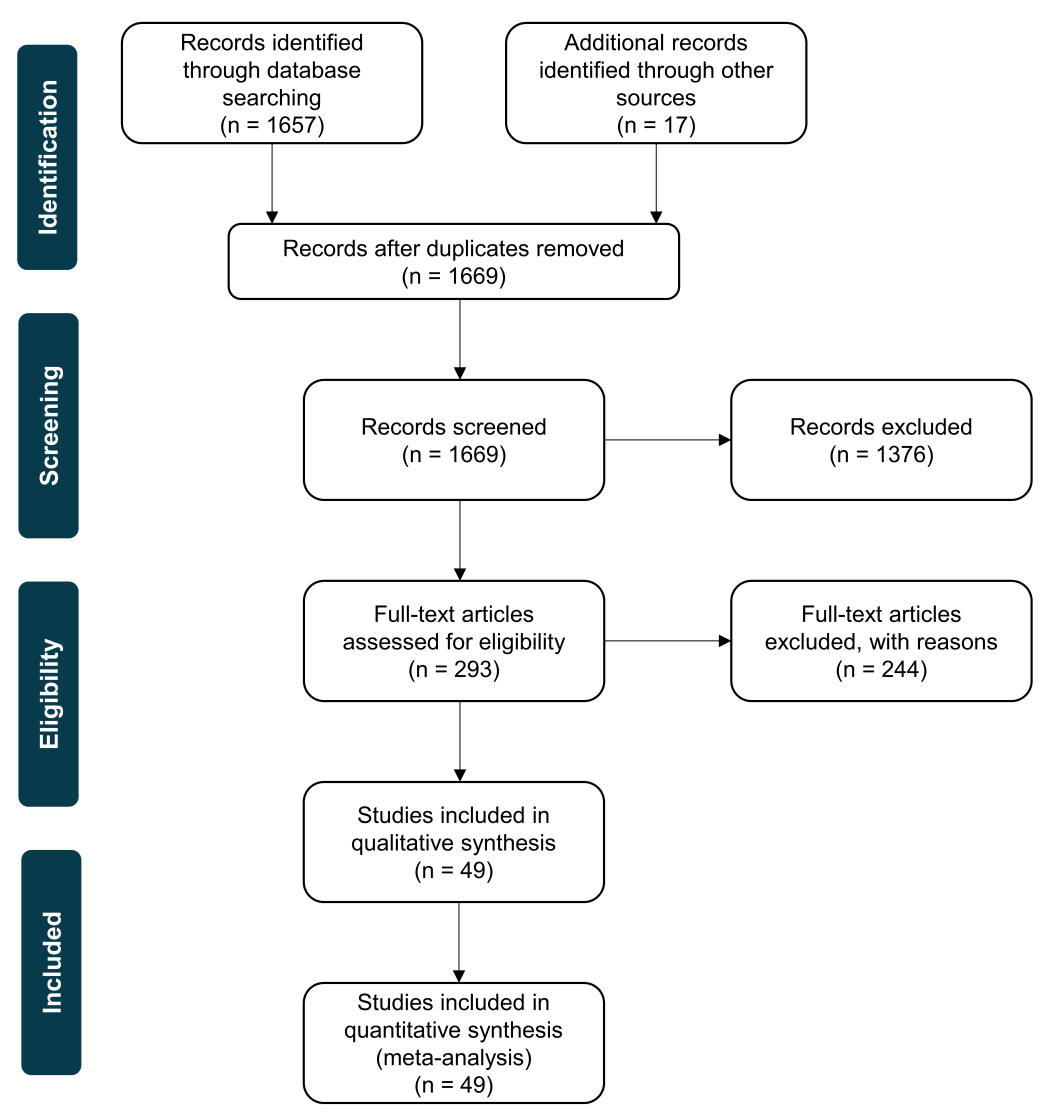

Figure 2: PRISMA diagram providing an overview of our review process. 


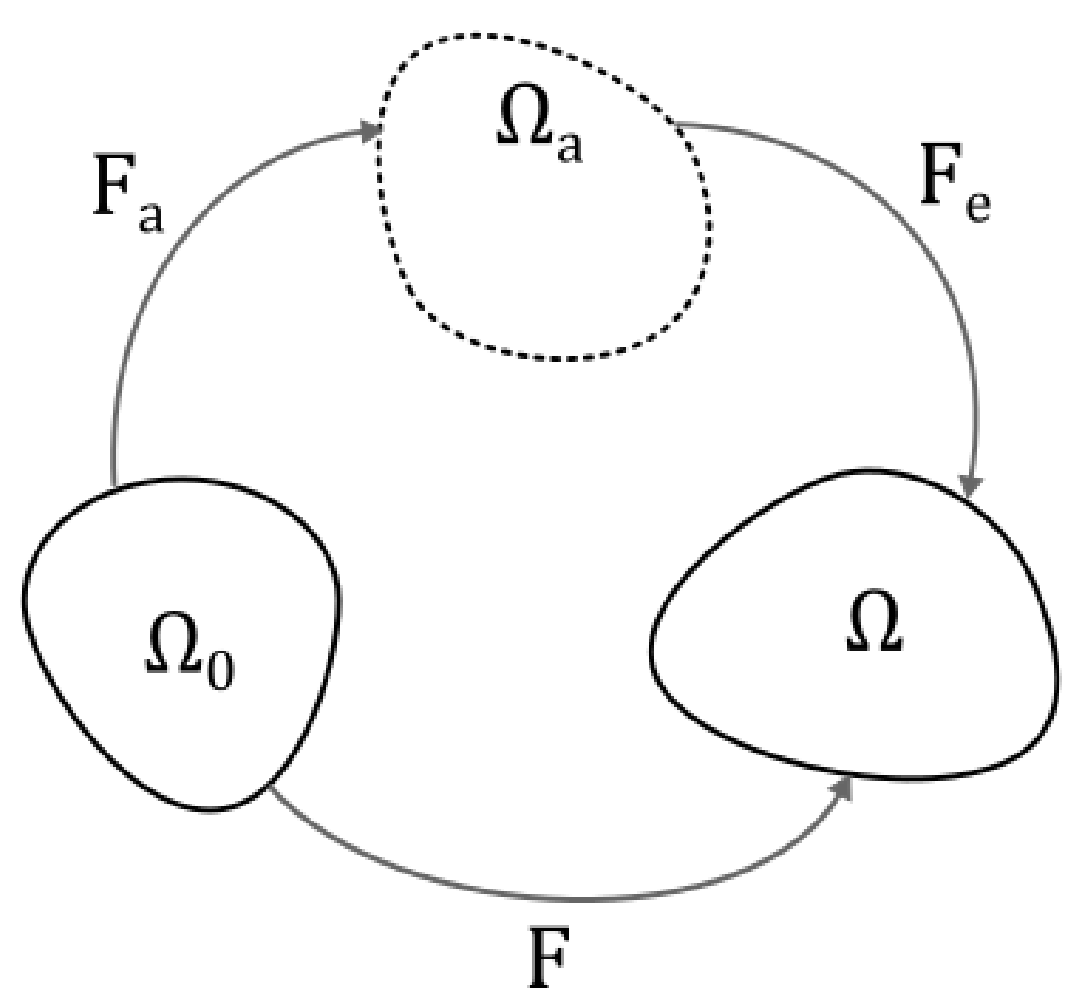

Figure 3: Illustration of the decomposition of the deformation gradient. 


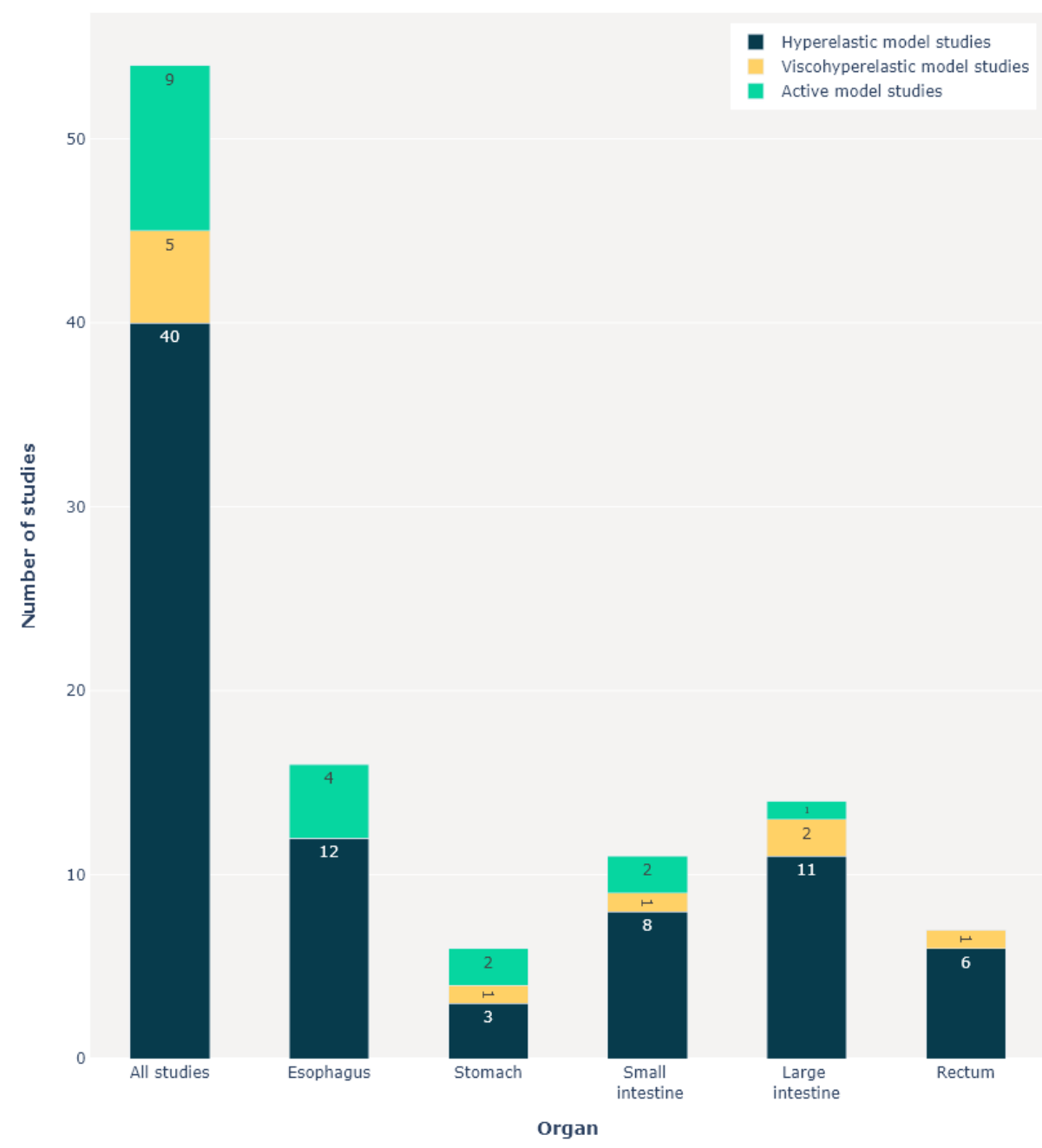

Figure 4: Organ-wise distribution of the studies with distinction between studies investigating hyperelastic, visco-hyperelastic, and active models. 


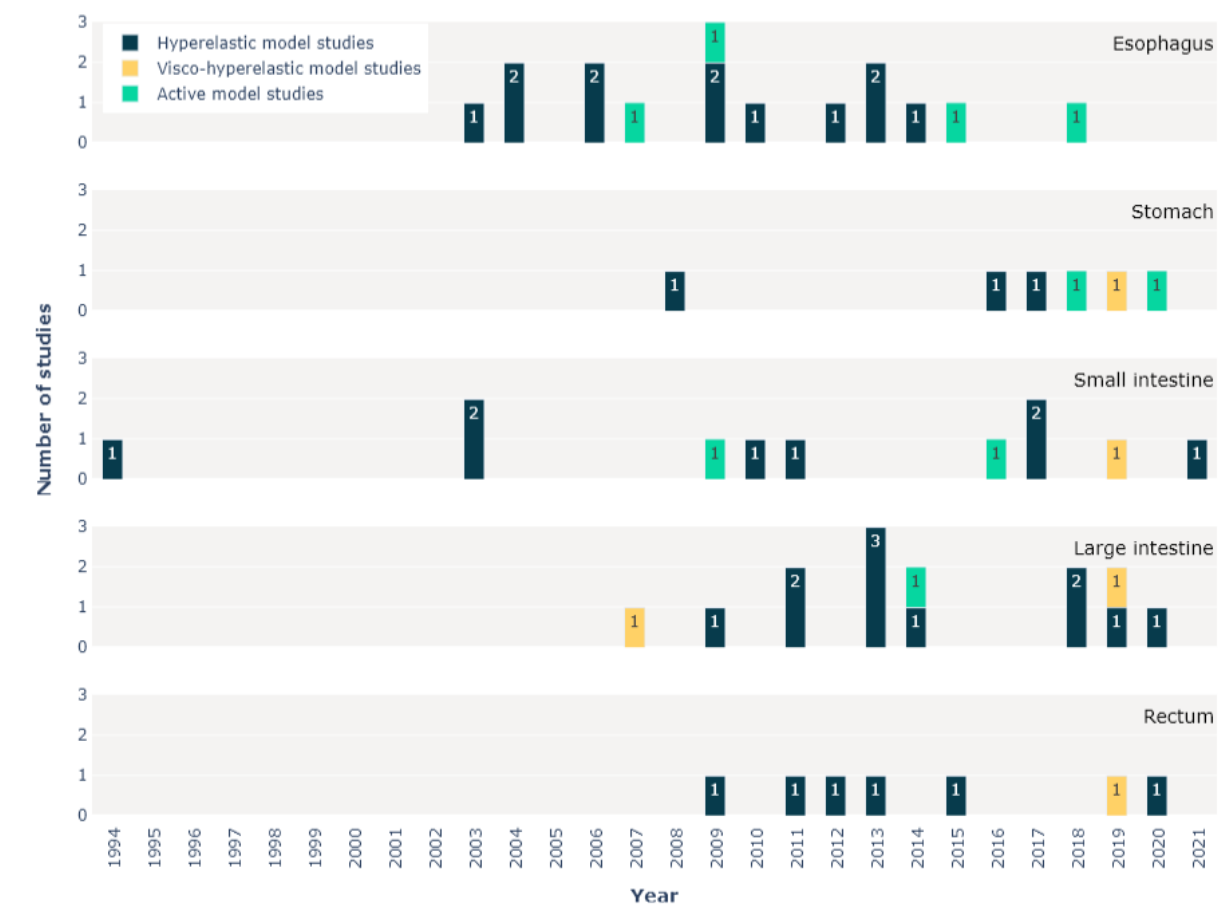

Figure 5: Organ-wise and year-wise distribution of the studies with distinction between studies investigating hyperelastic, visco-hyperelastic, and active models. 


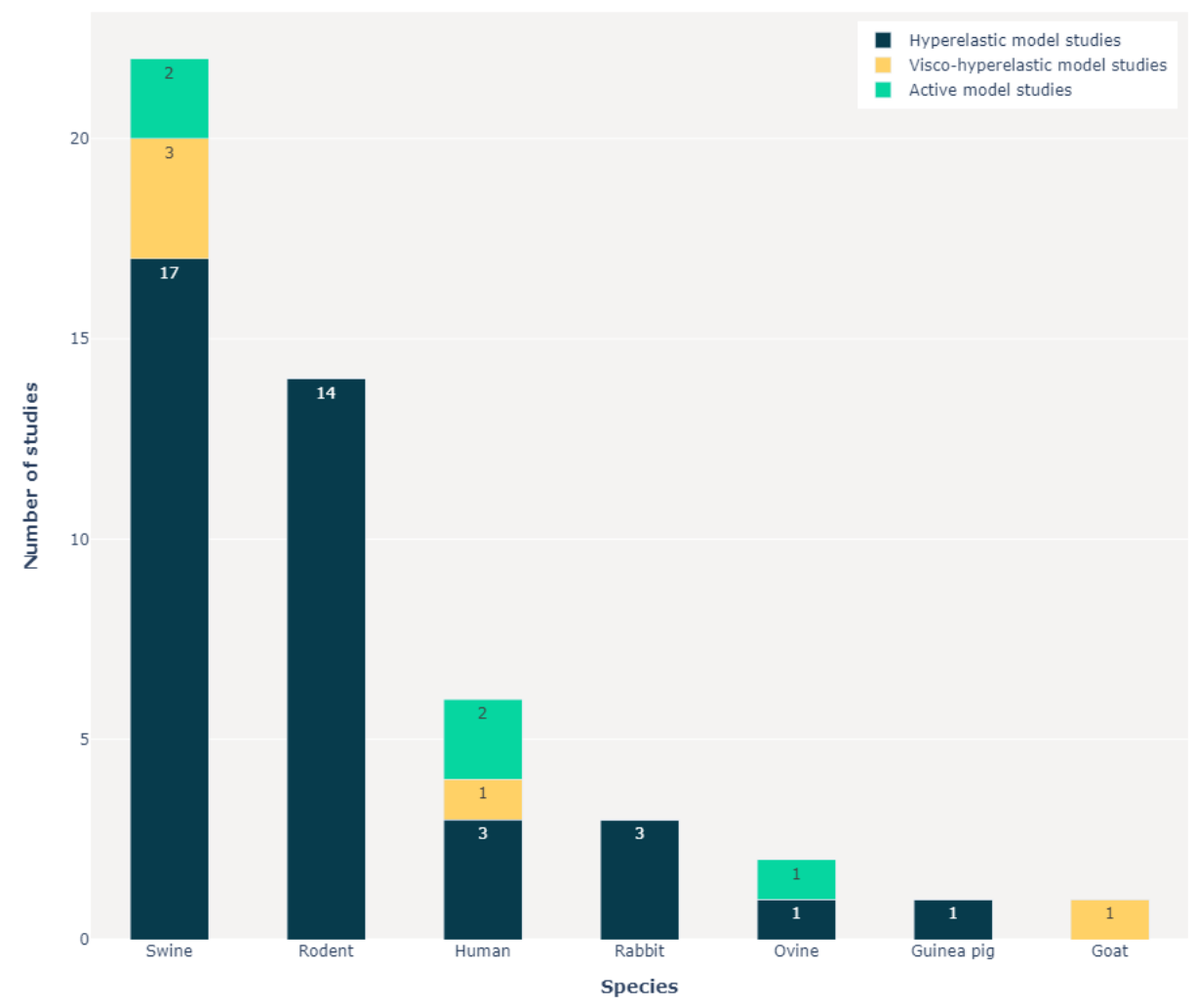

Figure 6: Organ-wise distribution of the studies with distinction of the tissue source used for mechanical testing. 


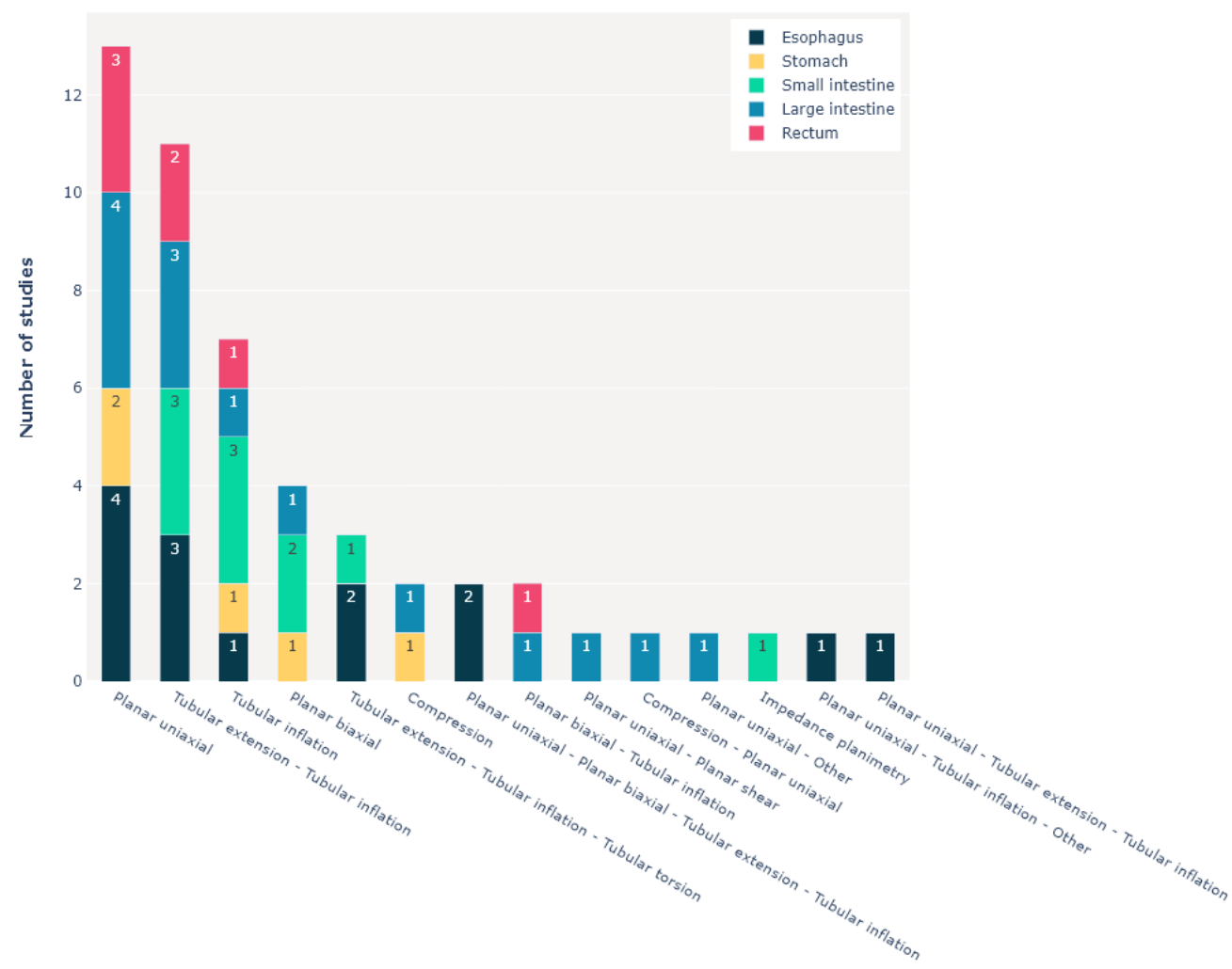

Figure 7: Organ-wise distribution of the studies with identification of the mechanical testing protocol utilized to estimate model parameters and/or validate the models. 


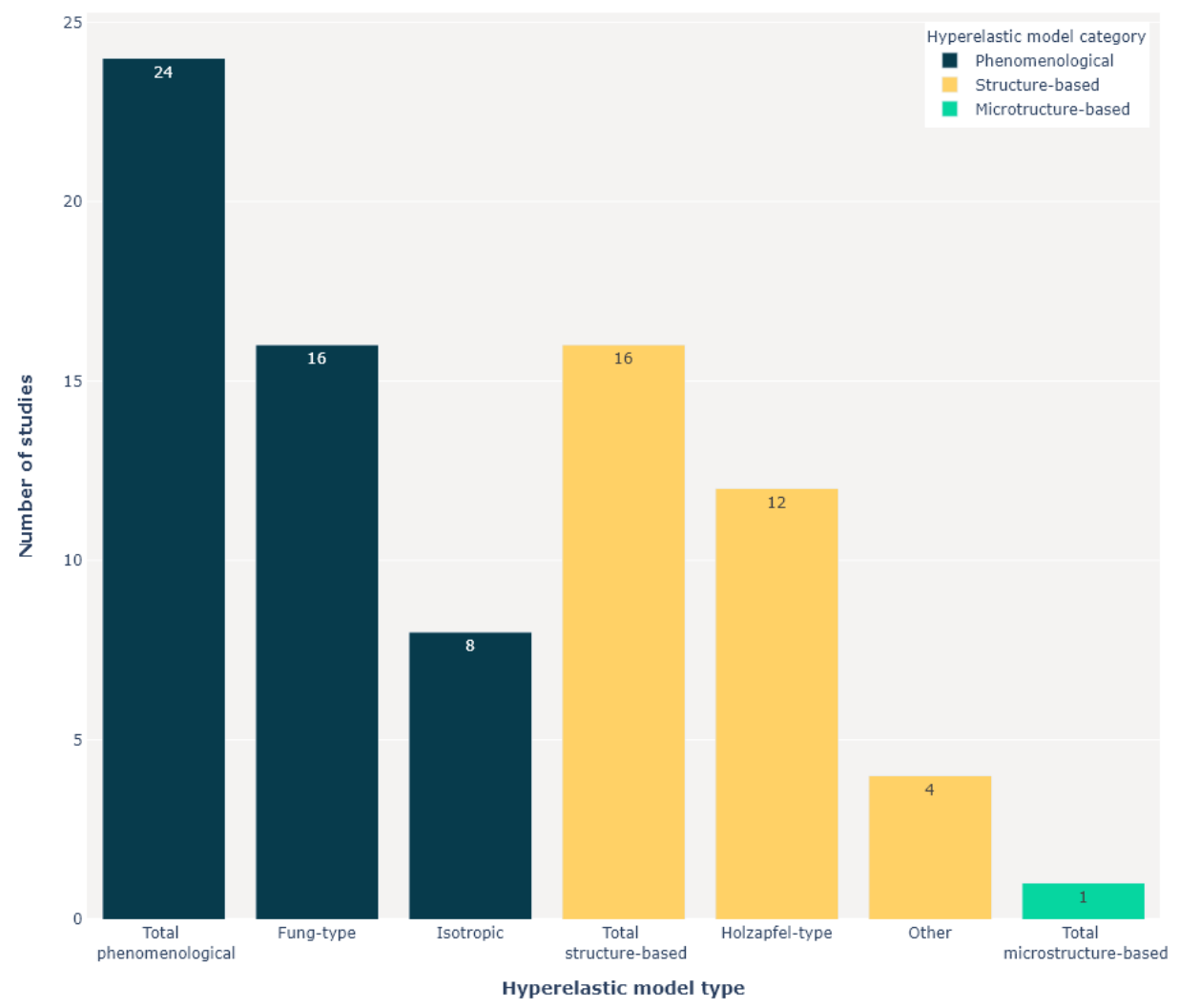

Figure 8: Distribution of the studies based on the hyperelastic model type proposed. 


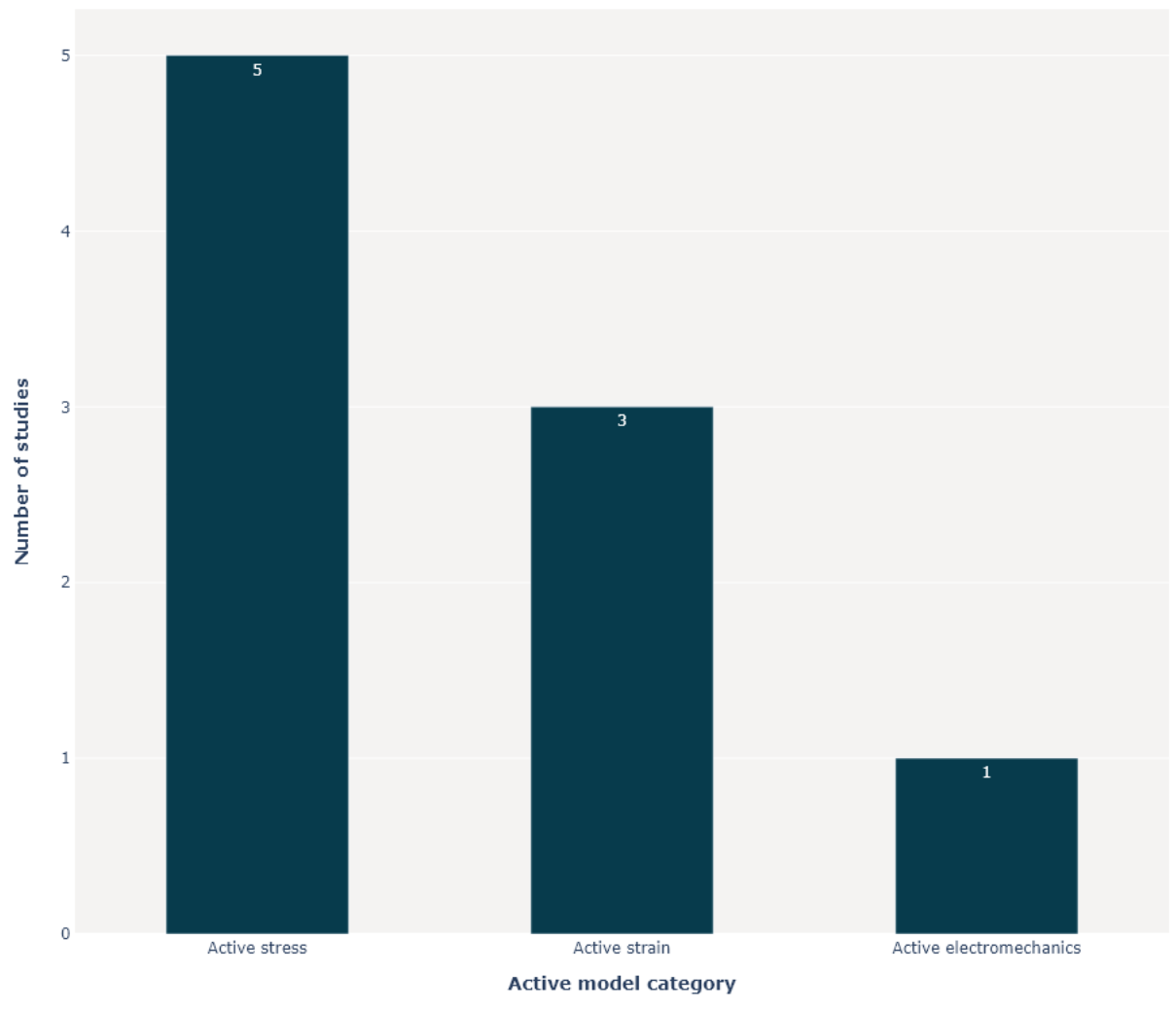

Figure 9: Distribution of the studies based on the active model type proposed. 